\title{
Statistics of ambiguous rotations
}

\author{
R. Arnold ${ }^{\mathrm{a}}$, P.E. Jupp ${ }^{\mathrm{b}, *}$, H. Schaeben ${ }^{\mathrm{c}}$ \\ ${ }^{a}$ School of Mathematics and Statistics, Victoria University of Wellington, P.O. Box 600, Wellington, New Zealand \\ ${ }^{b}$ School of Mathematics and Statistics, University of St. Andrews, St. Andrews KY16 9SS, UK \\ ${ }^{c}$ Geophysics and Geoscience Informatics, TU Bergakademie Freiberg, Germany
}

\begin{abstract}
The orientation of a rigid object can be described by a rotation that transforms it into a standard position. For a symmetrical object the rotation is known only up to multiplication by an element of the symmetry group. Such ambiguous rotations arise in biomechanics, crystallography and seismology. We develop methods for analyzing data of this form. A test of uniformity is given. Parametric models for ambiguous rotations are presented, tests of location are considered, and a regression model is proposed. An example involving orientations of diopside crystals (which have symmetry of order 2) is used throughout to illustrate how our methods can be applied.
\end{abstract}

Keywords: Frame, Orientation, Regression, Symmetric array, Symmetry, Test of location, Test of uniformity 2010 MSC: $62 \mathrm{H} 11,62 \mathrm{H} 15,62 \mathrm{H} 20$

\section{Introduction}

Data that are rotations of $\mathbb{R}^{3}$ occur in various areas of science, such as palaeo-magnetism $[25,36,46]$, plate tectonics and seismology [2, 16, 24, 44, 47], biomechanics [28, 39, 43], crystallography [15, 18], and texture analysis, i.e., analysis of orientations of crystalites [11, 26, 27]. The sample space is the 3-dimensional rotation group, $S O(3)$, and methods for handling such data are now an established part of directional statistics; see Mardia and Jupp [30, Section 13.2]. In some contexts the presence of symmetry means that the rotations are observed subject to ambiguity, so that it is not possible to distinguish a rotation $\mathbf{X}$ from $\mathbf{X R}$ for any rotation $\mathbf{R}$ in some given subgroup $K$ of $S O(3)$. From the mathematical point of view, the sample space is the quotient $S O(3) / K$ of $S O(3)$ by $K$. Such spaces arise in many scientific contexts: the case in which $K$ is generated by the rotations through $180^{\circ}$ about the coordinate axes gives the orthogonal axial frames considered by Arnold and Jupp [1], which can be used to describe aspects of earthquakes; several groups $K$ of low order occur as the symmetry groups of crystals; the icosahedral group is the symmetry group of some carborane molecules [20], of most closed-shell viruses [17], of the natural quasicrystal, icosahedrite [4], and of the blue phases of some liquid crystals [42, Section 6.1.2].

There are two very natural approaches to statistics on $S O(3) / K$ :

(i) the averaging approach, in which each point $[\mathbf{X}]=\{\mathbf{X R}: \mathbf{R} \in K\}$ of $S O(3) / K$ is regarded as the "average" of the $|K|$ points $\mathbf{X R}$, where $\mathbf{R}$ runs through $K$ and $|K|$ denotes the order of $K$;

(ii) the embedding approach, which uses a function $\mathbf{t}: S O(3) / K \rightarrow E$ to transform elements $[\mathbf{X}]$ of $S O(3) / K$ into vectors $\mathbf{t}([\mathbf{X}])$ in some inner-product space $E$.

A typical use of the averaging approach is the averaging of a probability density $f$ on $S O(3)$ to give a corresponding probability density $\bar{f}$ on $S O(3) / K$, defined by

$$
\bar{f}([\mathbf{X}])=|K|^{-1} \sum_{\mathbf{R} \in K} f(\mathbf{X R}) .
$$

\footnotetext{
*Corresponding author

Email addresses: richard. arnold@msor.vuw. ac.nz (R. Arnold), pej@st-andrews. ac.uk (P.E. Jupp), schaeben@tu-freiberg.de (H. Schaeben)
} 
Some simple algebra shows that every probability density on $S O(3) / K$ has the form (1) for some density $f$ on $S O(3)$ (which may be assumed to satisfy $f(\mathbf{X R})=f(\mathbf{X})$ for $\mathbf{R}$ in $K$ ). Given any family of distributions on $S O(3)$, the construction in (1) can be used (as in $[9,11,13,35]$ ) to produce a corresponding family of distributions on $S O(3) / K$. As the construction in (1) is a special case of forming mixtures, the latter family is very simple in form but has the disadvantage that inference can be rather cumbersome and quite demanding numerically. The embedding approach of mapping the sample space into some vector space is already used to very good effect in (i) directional statistics (see, e.g., [30, Section 10.8]), (ii) landmark shape (see, e.g., [10]). The major advantages of the embedding approach are that (i) it focuses attention on suitable transforms of the data, (ii) standard Euclidean intuition can be used, (iii) it leads naturally to the use of exponential models, a class for which inference is straight-forward, (iv) summary statistics, tests of standard hypotheses, and regression models, etc., can be constructed in a simple and appealing way using the transforms.

Almost all publications (notably $[9,11,13,31,35,47]$ ) on methodology for analyzing ambiguous rotations have taken the averaging approach. It seems that the only paper taking the embedding approach is that by Arnold and Jupp [1] for the orthorhombic case, $K=D_{2}$. For rotations of the plane that are ambiguous in the sense that rotation through angle $\theta$ is equivalent to rotation through $\theta+\pi$, the standard technique is "doubling the angles", replacing $\theta$ by $2 \theta$ [30, p. 1]. This is equivalent to using the embedding $\theta \mapsto(\cos (2 \theta), \sin (2 \theta))$ of the circle into the plane.

The object of this paper is to give a unified account of some general basic tools, based on the embedding approach, for the analysis of data consisting of ambiguous rotations with a finite symmetry group. The tools that we present here provide a first step towards the analysis of data on ambiguous rotations that arise in complicated contexts. Refinements of these tools that are needed for the analysis of data with, e.g., a spatial element, will be considered in subsequent work.

In Section 2 we recall the traditional representation of the orientations of rigid objects by rotations. For symmetric objects, these rotations are ambiguous, being known only up to post-multiplication by members of the symmetry group. We then introduce an alternative representation in terms of frames, i.e., sets of vectors or axes in $\mathbb{R}^{3}$. For symmetric objects, the ambiguity in these frames is removed by passing to appropriate equivalence classes, the symmetric frames. Section 3 introduces simple transformations that embed spaces of symmetric frames into vector spaces of symmetric arrays, thus enabling the use of standard vector operations and Euclidean intuition. These transformations are the backbone of the embedding approach used in this paper. A first use, given in Sections 3.2-3.3, is the definition of sample mean and dispersion. Section 4 provides a simple statistic for testing uniformity and gives its large-sample asymptotic distribution. This test is complemented by consistent tests based on the averaging approach. Section 5 considers a general class of distributions on $S O(3) / K$ that are parameterized by a location parameter and concentration parameter. Particular attention is given to the curved exponential models in this class and their high-concentration limits are given. In Section 6 one- and two-sample tests of location are presented. Section 7 considers paired data: some tests of independence are proposed, a regression model is presented, and the concept of misorientation (used in crystallography and texture analysis) is given a quantitative form. Section 8 contains some concluding remarks. The Appendix gives some outline proofs and calculations.

\section{Ambiguous rotations}

\subsection{Symmetry groups}

The usual way of describing the orientation of a rigid object in $\mathbb{R}^{3}$ is by a rotation that transforms it into some standard orientation. If the object is asymmetrical then this rotation is unique, so that the orientations of the object correspond to elements of the group, $S O(3)$, of rotations of $\mathbb{R}^{3}$. If the object is symmetrical then the set of rotations that have no visible effect on the object forms a subgroup $K$ of $S O(3)$. Then the orientations of the object correspond to elements of the homogeneous space $S O(3) / K$, i.e., the set of equivalence classes of elements of $S O(3)$ under the right action of $K$. We shall consider the cases in which $K$ is finite. In particular, the orientations of T-shaped, X-shaped and +-shaped objects in $\mathbb{R}^{3}$ are elements of $S O(3) / K$ with $K=C_{2}, D_{2}$ and $D_{4}$, respectively. For $\mathbf{U}$ in $S O(3)$ we shall denote the equivalence class $\{\mathbf{U R}: \mathbf{R} \in K\}$ of $\mathbf{U}$ in $S O(3) / K$ by [U].

The finite subgroups of $S O(3)$ are known also as the point groups of the first kind. The classification result for these groups, given, e.g., in [32, Section 2.4], states that any such group is isomorphic to one of the following: the cyclic groups, $C_{r}$, for $r \in\{1,2, \ldots\}$, the dihedral groups, $D_{r}$, for $r \in\{2,3, \ldots\}$, the tetrahedral group, $T$, the octahedral 


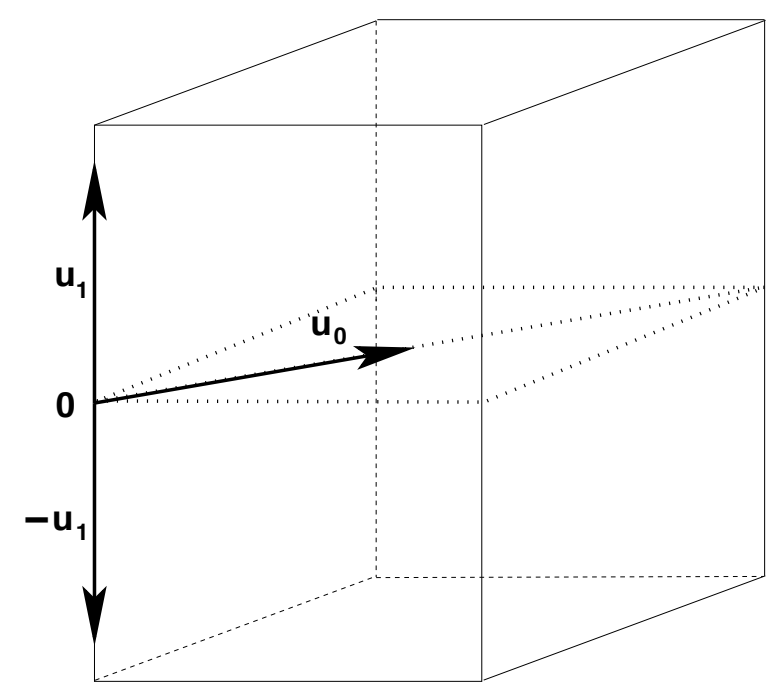

Figure 1: Orientation of a monoclinic crystal. $\mathbf{u}_{0}$ and $\mathbf{u}_{1}$ are orthogonal unit vectors.

group, $O$, and the icosahedral group, $Y$. These groups are listed in Table 1, together with the frames of vectors that will be used to represent elements of the sample spaces $S O(3) / K$. The group $C_{1}$ has one element, the identity, $\mathbf{I}_{3}$.

The simplest non-trivial group is $C_{2}$. Objects with this symmetry group include monoclinic crystals, such as those of the mineral, diopside. The orientation of a monoclinic crystal can be specified by $\left(\mathbf{u}_{0}, \pm \mathbf{u}_{1}\right)$, where $\mathbf{u}_{0}$ and $\mathbf{u}_{1}$ are orthogonal unit vectors; see Figure 1 and the row for $C_{2}$ in Table 1. Figure 2 shows a sample of orientations $\left(\mathbf{u}_{0}, \pm \mathbf{u}_{1}\right)$ of 100 crystals of diopside. The directions $\mathbf{u}_{0}$ are shown as triangles, and the axes $\pm \mathbf{u}_{1}$ as circles. We shall use this data set (and a similar two-sample data set) repeatedly to illustrate our methods.

\subsection{Frames and symmetric frames}

A more basic way of describing the orientation of an asymmetrical rigid object than by a rotation is by a frame, meaning a set of vectors or axes in $\mathbb{R}^{3}$. It is more basic in that it does not require the choice of a standard orientation. Similarly, a more basic way of describing the orientation of a rigid object with symmetry group $K$ than by an ambiguous rotation is by a $K$-frame, i.e., an equivalence class of frames. For $K=C_{r}$ with $r \geq 3$ or $K=D_{r}$ with $r \geq 3$, it is convenient to take the vectors of the frame to be unit normals to the sides of a regular $r$-gon; for $K=C_{2}$ we take a unit vector and an axis orthogonal to it; for $K=D_{2}$ we take a pair of orthogonal axes; for $K=T, O$ or $Y$, it is convenient to take the vectors to be unit normals to the sides of a regular tetrahedron, cube or dodecahedron, respectively. Permutation of the vectors of the frame by the action of $K$ leads to ambiguity. This ambiguity is removed by passing to the corresponding $K$-frame, i.e., the equivalence class of the frame under such permutations. The $K$-frames will be denoted by square brackets, e.g., for $K=C_{r},\left[\mathbf{u}_{1}, \ldots, \mathbf{u}_{r}\right]$ denotes the $K$-frame arising from $\left(\mathbf{u}_{1}, \ldots, \mathbf{u}_{r}\right)$. By a symmetric frame, we shall mean a $K$-frame for some $K$. The frames that we consider are listed in Table 1 , together with an indication of the ambiguities. For notational simplicity, we shall sometimes abuse language and denote a $K$-frame by $[\mathbf{U}]$.

Special cases of the symmetries listed in Table 1 arise in the 7 crystal systems: triclinic, monoclinic, trigonal, tetragonal, orthorhombic, hexagonal and cubic with symmetry groups $C_{1}, C_{2}, C_{3}, C_{4}, D_{2}, D_{6}$ and $O$, respectively.

\section{Transforming symmetric frames to symmetric arrays}

\subsection{Embeddings of the sample spaces}

In order to carry out statistics on $S O(3) / K$, we shall take the embedding approach.

The embedding will be a well-defined equivariant one-to-one function $\mathbf{t}: S O(3) / K \rightarrow E$, where $E$ is an innerproduct space on which $S O(3)$ acts, such that $\mathbf{t}([\mathbf{U}])$ has expectation $\mathbf{0}$ if $[\mathbf{U}]$ is uniformly distributed on $S O(3) / K$. For $E=L^{2}\{S O(3)\}$, the space of square-integrable functions on $S O(3)$, a very wide class of such embeddings can be 


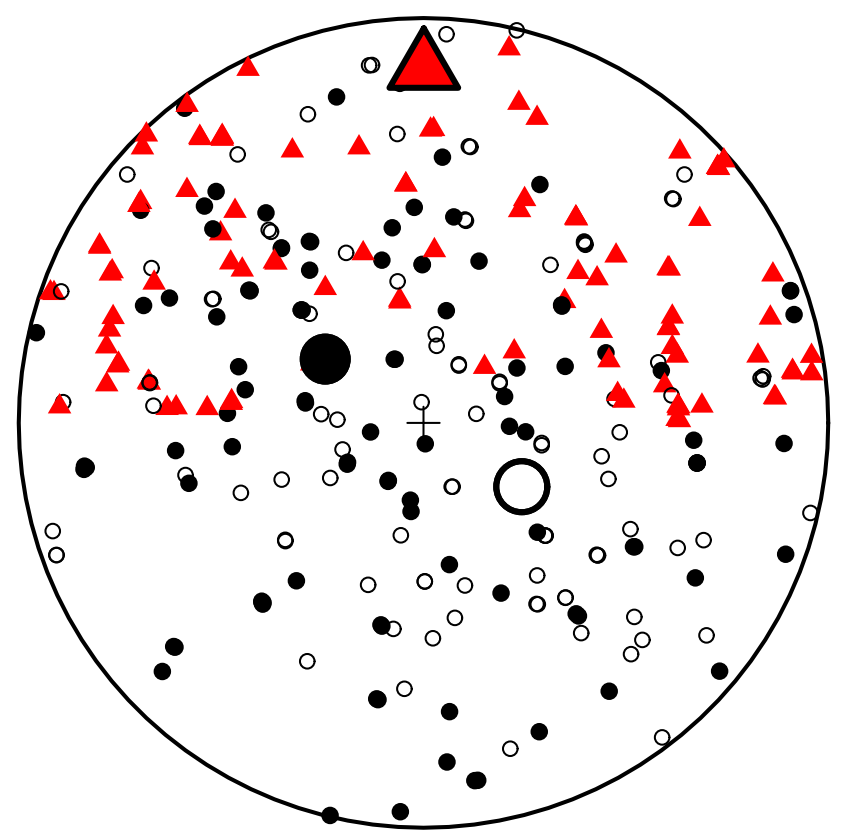

Figure 2: $C_{2}$ symmetry. Stereonet of $\mathbf{u}_{0}$ vectors, shown as red triangles, and $\pm \mathbf{u}_{1}$ axes, shown as circles, given by orientations of 100 diopside crystals. The disc is a stereographic projection of these vectors and axes, showing the whole of the sphere, so that each axis appears twice with filled circles denoting the lower ends of axes and open circles the upper ends. The sample mean is shown in large symbols.

obtained by averaging over $K$. Let $\mathbf{t}_{0}: S O(3) \rightarrow L^{2}\{S O(3)\}$ be an embedding of the type used in the Hilbert space approach [30, Sections 10.8, 13.2.2] to Sobolev tests of uniformity; see Giné [12] and Prentice [37, Section 4]. Define $\mathbf{t}: S O(3) / K \rightarrow L^{2}\{S O(3)\}$ by

$$
\mathbf{t}([\mathbf{U}])=|K|^{-1} \sum_{\mathbf{R} \in K} \mathbf{t}_{0}(\mathbf{U R}),
$$

where $|K|$ denotes the number of elements in $K$. If $\mathbf{t}$ is one-to-one then it is an embedding. In general, such $\mathbf{t}$ are quite complicated, so in this paper, for each $K$, we focus on a simple choice of embedding, $\mathbf{t}_{K}$, of $S O(3) / K$ into an appropriate space of symmetric arrays. These $\mathbf{t}_{K}$ are given in Table 2 and are based on symmetric $r$-way arrays, $\otimes^{r} \mathbf{u}_{i}$, which can be thought of as " $r$ th powers" of vectors. (In mathematical terms, they are coordinate representations of $r$-fold tensor products.) For vectors $\mathbf{u}_{1}, \ldots, \mathbf{u}_{r}$ with $\mathbf{u}_{i}=\left(u_{i, 1}, u_{i, 2}, u_{i, 3}\right)^{\top}$ for $i \in\{1, \ldots, r\}$, the $r$-way array $\otimes^{r} \mathbf{u}_{i}$ has $\left(j_{1}, \ldots, j_{r}\right)$ th entry

$$
\left(\otimes^{r} \mathbf{u}_{i}\right)_{j_{1}, \ldots, j_{r}}=\prod_{k=1}^{r} u_{i, j_{k}}, \quad 1 \leq j_{1}, \ldots, j_{r} \leq 3,
$$

e.g., $\otimes^{2} \mathbf{u}_{i}=\mathbf{u}_{i} \mathbf{u}_{i}^{\top}$. Some of the $\mathbf{t}_{K}$ involve also the arrays symm $\left(\otimes^{r / 2} \mathbf{I}_{3}\right)$ for $r$ even. These have entries

$$
\left\{\operatorname{symm}\left(\otimes^{r / 2} \mathbf{I}_{3}\right)\right\}_{j_{1}, \ldots, j_{r}}=\frac{1}{r !} \sum_{\sigma \in \Sigma_{r}} \prod_{i=1}^{r / 2} \delta_{\sigma\left(j_{2 i-1}\right) \sigma\left(j_{2 i}\right)},
$$

where $\Sigma_{r}$ is the group of permutations of $\{1, \ldots, r\}$ and $\delta_{i j}=1$ for $i=j, \delta_{i j}=0$ otherwise. Thus, e.g.,

$$
\operatorname{symm}\left(\otimes^{2} \mathbf{I}_{3}\right)_{j_{1} j_{2} j_{3} j_{4}}=\left(\delta_{j_{1} j_{2}} \delta_{j_{3} j_{4}}+\delta_{j_{1} j_{3}} \delta_{j_{2} j_{4}}+\delta_{j_{1} j_{4}} \delta_{j_{2} j_{3}}\right) / 3 \text {. }
$$

For many of the tests and other objects that we discuss below it is not necessary to construct the representations $\mathbf{t}_{K}([\mathbf{U}])$ explicitly; it is sufficient to compute inner products $\left\langle\mathbf{t}_{K}([\mathbf{U}]), \mathbf{t}_{K}([\mathbf{V}])\right\rangle$ as required, where $\langle\cdot, \cdot\rangle$ is the standard inner product on the relevant space of symmetric arrays. The $\left\langle\mathbf{t}_{K}([\mathbf{U}]), \mathbf{t}_{K}([\mathbf{V}])\right\rangle$ are given in Table 3. 
Table 1: Symmetry groups and frames.

\begin{tabular}{|c|c|c|c|c|}
\hline Group, $K$ & Name & $|K|$ & Frame & \\
\hline$C_{1}$ & trivial & 1 & $\left(\mathbf{u}_{1}, \mathbf{u}_{2}, \mathbf{u}_{3}\right)$ & $\mathbf{u}_{1}, \mathbf{u}_{2}, \mathbf{u}_{3}$ orthonormal, $\mathbf{u}_{3}=\mathbf{u}_{1} \times \mathbf{u}_{2}$ \\
\hline$C_{2}$ & cyclic & 2 & $\left(\mathbf{u}_{0}, \pm \mathbf{u}_{1}\right)$ & $\mathbf{u}_{0}, \mathbf{u}_{1}$ orthonormal \\
\hline$C_{r} \quad(r \geq 3)$ & cyclic & $r$ & $\left(\mathbf{u}_{1}, \ldots, \mathbf{u}_{r}\right)$ & $\begin{array}{l}\mathbf{u}_{1}, \ldots, \mathbf{u}_{r} \text { coplanar, } \\
\text { known up to cyclic order, } \\
\mathbf{u}_{i}^{\top} \mathbf{u}_{i-1}=\cos (2 \pi / r) \text { for } i \in\{2, \ldots, r\}\end{array}$ \\
\hline$D_{2}$ & dihedral & 4 & $\left( \pm \mathbf{u}_{1}, \pm \mathbf{u}_{2}\right)$ & orthogonal axes \\
\hline$D_{r} \quad(r \geq 3)$ & dihedral & $2 r$ & $\left(\mathbf{u}_{1}, \ldots, \mathbf{u}_{r}\right)$ & $\begin{array}{l}\mathbf{u}_{1}, \ldots, \mathbf{u}_{r} \text { coplanar, } \\
\text { known up to cyclic order and reversal, } \\
\mathbf{u}_{i}^{\top} \mathbf{u}_{i-1}=\cos (2 \pi / r) \text { for } i \in\{2, \ldots, r\}\end{array}$ \\
\hline$T=A_{4}$ & tetrahedral & 12 & $\left\{\mathbf{u}_{1}, \ldots, \mathbf{u}_{4}\right\}$ & $\mathbf{u}_{i}^{\top} \mathbf{u}_{j}=-1 / 3$ for $i \neq j$ \\
\hline$O=\Sigma_{4}$ & $\begin{array}{l}\text { octahedral } \\
=\text { cubic }\end{array}$ & 24 & $\left\{ \pm \mathbf{u}_{1}, \pm \mathbf{u}_{2}, \pm \mathbf{u}_{3}\right\}$ & orthogonal axes \\
\hline$Y=A_{5}$ & $\begin{array}{l}\text { icosahedral } \\
=\text { dodecahedral }\end{array}$ & 60 & $\left\{ \pm \mathbf{u}_{1}, \ldots, \pm \mathbf{u}_{6}\right\}$ & $\left|\mathbf{u}_{i}^{\top} \mathbf{u}_{j}\right|=5^{-1 / 2}$ for $i \neq j$ \\
\hline
\end{tabular}

The $\mathbf{u}_{i}$ are unit vectors; $|K|$ is the number of elements in $K$.

Define $\rho^{2}$ by

$$
\rho^{2}=\|\mathbf{t}([\mathbf{U}])\|^{2},
$$

which has the same value for all $\mathbf{U}$ in $S O(3)$. Then $\mathbf{t}$ embeds $S O(3) / K$ in the sphere of radius $\rho$ with centre the origin in the vector space $E$. Values of $\rho^{2}$ are given in Table 4.

Each symmetric frame can be represented by an element $\mathbf{U}$ of $S O(3)$. In the triclinic case, where $K=C_{1}$, $\mathbf{U}$ is unique and $\mathbf{t}([\mathbf{U}])=\mathbf{U}$. We have restricted our attention to point groups, $K$, of the first kind, i.e., excluding reflections. However, in situations where reflection symmetries are also present we can adopt a right-handed convention for all orientations, and then neglect reflections. For example, we can treat observations on $O(3) /\left\{\mathbf{I}_{3},-\mathbf{I}_{3}\right\}$ (where $O(3)$ denotes the group of orthogonal transformations of $\mathbb{R}^{3}$ ) in the same way as those on $S O(3)=S O(3) / C_{1}$.

\subsection{Sample mean}

Observations $\left[\mathbf{U}_{1}\right], \ldots,\left[\mathbf{U}_{n}\right]$ in $S O(3) / K$ can usefully be summarized by the sample mean $\overline{\mathbf{t}}$ of their images by $\mathbf{t}$, i.e., by $\overline{\mathbf{t}}=\sum_{k=1}^{n} \mathbf{t}\left(\left[\mathbf{U}_{k}\right]\right) / n$. The sample mean $[\overline{\mathbf{U}}]$ is defined as the $[\mathbf{U}]$ in $S O(3) / K$ that maximizes $\langle\mathbf{t}([\mathbf{U}]), \overline{\mathbf{t}}\rangle$. Although $[\overline{\mathbf{U}}]$ is not necessarily unique, it follows from [3, Theorem 3.2] that if $\left[\mathbf{U}_{1}\right], \ldots,\left[\mathbf{U}_{n}\right]$ are generated by a continuous distribution then $[\overline{\mathbf{U}}]$ is unique with probability 1 . If $K=C_{2}$ then the sample mean of $\left[\mathbf{U}_{1}\right], \ldots,\left[\mathbf{U}_{n}\right]$ is

$$
\overline{\mathbf{t}}=\left(n^{-1} \sum_{k=1}^{n} \mathbf{u}_{k 0}, n^{-1} \sum_{k=1}^{n} \mathbf{u}_{k 1} \mathbf{u}_{k 1}^{\top}-(1 / 3) \mathbf{I}_{3}\right),
$$

where $\left[\mathbf{U}_{k}\right]$ is represented by the $C_{2}$-frame $\left(\mathbf{u}_{k 0}, \pm \mathbf{u}_{k 1}\right)$ for $k \in\{1, \ldots, n\}$. For general $K$, there does not appear to be an explicit expression for [ $\overline{\mathbf{U}}]$. In the cases $K=C_{r}$ (with $r \geq 2$ ) or $D_{r}$ (with $r \geq 2$ ) there are the following explicit recipes for an approximation to $[\overline{\mathbf{U}}]$.

For $r=2$, let $\left(\mathbf{u}_{10}, \pm \mathbf{u}_{11}\right), \ldots,\left(\mathbf{u}_{n 0}, \pm \mathbf{u}_{n 1}\right)$ or $\left( \pm \mathbf{u}_{11}, \pm \mathbf{u}_{12}\right), \ldots,\left(\mathbf{u}_{n 1}, \pm \mathbf{u}_{n 2}\right)$ be symmetric frames representing $n$ elements of $S O(3) / C_{2}$ or $S O(3) / D_{2}$, respectively. For $r \geq 3$, let $\left[\mathbf{u}_{11}, \ldots, \mathbf{u}_{1 r}\right], \ldots,\left[\mathbf{u}_{n 1}, \ldots, \mathbf{u}_{n r}\right]$ be symmetric frames representing $n$ elements of $S O(3) / C_{r}$ or $S O(3) / D_{r}$.

The first step is to estimate the $K$-invariant axis. For $K=D_{2}$, define $\pm \mathbf{u}_{i 0}$ by $\pm \mathbf{u}_{i 0}= \pm \mathbf{u}_{i 1} \times \mathbf{u}_{i 2}$ for $i \in\{1, \ldots, n\}$. For $r \geq 3$, define $\mathbf{u}_{i 0}$ by $\mathbf{u}_{i 0}=\{\sin (2 \pi / r)\}^{-1} \mathbf{u}_{i 1} \times \mathbf{u}_{i 2}, i \in\{1, \ldots, n\}$. In the case $K=C_{r}$ with $r \geq 2$, define $\tilde{\mathbf{u}}_{0}$ to be the mean direction of the unit vectors $\mathbf{u}_{10}, \ldots, \mathbf{u}_{n 0}$, i.e., the unit vector that is a positive multiple of $\sum_{i=1}^{n} \mathbf{u}_{i 0}$. In the case $K=D_{r}$ with $r \geq 2$, define the axis $\pm \tilde{\mathbf{u}}_{0}$ to be the mean of the axes $\pm \mathbf{u}_{10}, \ldots, \pm \mathbf{u}_{n 0}$, i.e., the dominant principal axis of $\sum_{i=1}^{n} \mathbf{u}_{i 0} \mathbf{u}_{i 0}^{\top}$. Choose orthonormal vectors $\mathbf{e}_{1}$ and $\mathbf{e}_{2}$ that are normal to $\tilde{\mathbf{u}}_{0}$. 


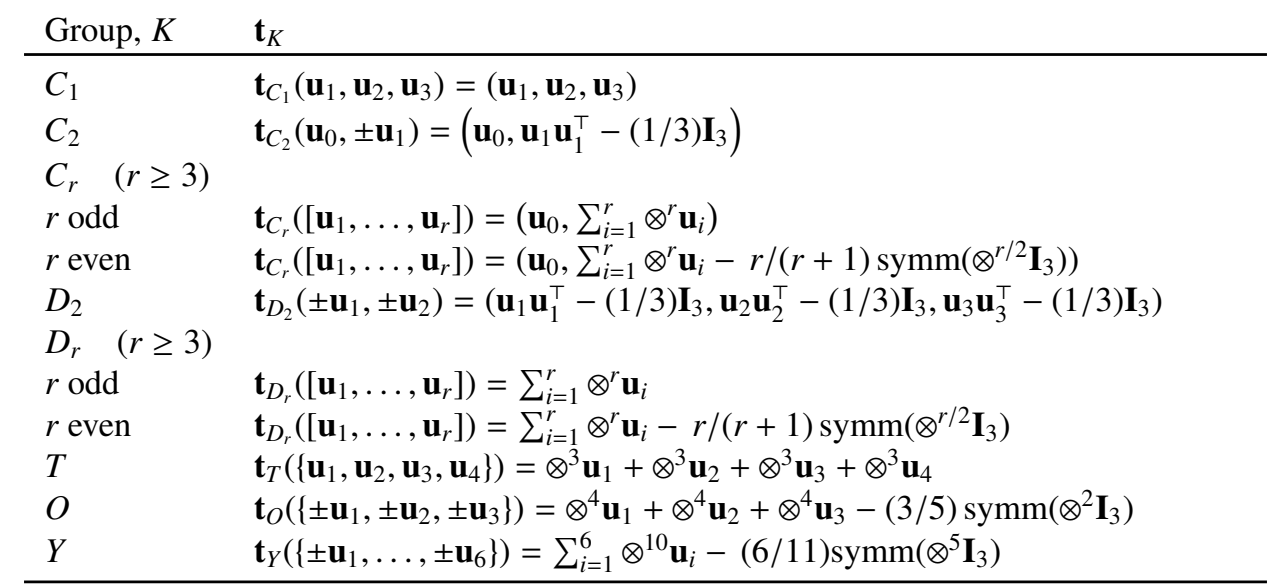

For $C_{r}$ with $r \geq 3, \mathbf{u}_{0}=\{\sin (2 \pi / r)\}^{-1} \mathbf{u}_{1} \times \mathbf{u}_{2}$. For $D_{2}, \mathbf{u}_{3}= \pm \mathbf{u}_{1} \times \mathbf{u}_{2}$. The symmetric arrays $\otimes^{r} \mathbf{u}_{i}$ and $\operatorname{symm}\left(\otimes^{r / 2} \mathbf{I}_{3}\right)$ are defined in (2) and (3), respectively.

For $i \in\{1, \ldots, n\}$, define $\theta_{i 1}, \ldots, \theta_{i r}$ by

$$
\left\|\left(\mathbf{I}_{3}-\tilde{\mathbf{u}}_{0} \tilde{\mathbf{u}}_{0}^{\top}\right) \mathbf{u}_{i j}\right\|^{-1}\left(\mathbf{I}_{3}-\tilde{\mathbf{u}}_{0} \tilde{\mathbf{u}}_{0}^{\top}\right) \mathbf{u}_{i j}=\cos \left(\theta_{i j}\right) \mathbf{e}_{1}+\sin \left(\theta_{i j}\right) \mathbf{e}_{2}
$$

for $j \in\{1, \ldots, r\}$. Define $\hat{\theta}$ as the angle in $[0,2 \pi / r)$ such that $r \hat{\theta}$ is the mean direction of $r \theta_{11}, \ldots, r \theta_{1}, \ldots, r \theta_{n 1}, \ldots, r \theta_{n r}$, and put $\tilde{\mathbf{u}}_{j}=\cos (\hat{\theta}+j 2 \pi / r) \mathbf{e}_{1}+\sin (\hat{\theta}+j 2 \pi / r) \mathbf{e}_{2}$ for $j \in\{1, \ldots, r\}$. Then $\left(\tilde{\mathbf{u}}_{0}, \pm \tilde{\mathbf{u}}_{1}\right)$ (for $K=C_{2}$ and $\left.r=2\right)$ or $\left( \pm \tilde{\mathbf{u}}_{1}, \pm \tilde{\mathbf{u}}_{2}\right)$ (for $K=D_{2}$ and $r=2$ ) or $\left[\tilde{\mathbf{u}}_{1}, \ldots, \tilde{\mathbf{u}}_{r}\right]$ (for $r \geq 3$ ) is an approximation to the sample mean. If the directions $\mathbf{u}_{10}, \ldots, \mathbf{u}_{n 0}$ (for $S O(3) / C_{r}$ ) or the axes $\pm \mathbf{u}_{10}, \ldots, \pm \mathbf{u}_{n 0}$ (for $S O(3) / D_{r}$ ) are concentrated then with high probability the above ap-

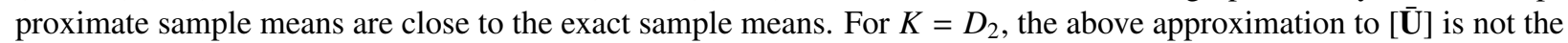
same as that given in Arnold and Jupp [1, Section 2.2].

\subsection{Sample dispersion}

A sensible measure of dispersion is

$$
d=\rho^{2}-\|\overline{\mathbf{t}}\|^{2}
$$

analogous to the quantity $1-\bar{R}^{2}$ used for spherical data; see Mardia and Jupp [30, p. 164]. The dispersion satisfies the inequalities $0 \leq d \leq \rho^{2}$, where $\rho^{2}$ is defined in (4). Since $\mathbf{t}$ is one-to-one, $d=0$ if and only if $\left[\mathbf{U}_{1}\right]=\cdots=$ $\left[\mathbf{U}_{n}\right]$. Transformation of $\left[\mathbf{U}_{1}\right], \ldots,\left[\mathbf{U}_{n}\right]$ to $\left[\mathbf{V} \mathbf{U}_{1}\right], \ldots,\left[\mathbf{V} \mathbf{U}_{n}\right]$ with $\mathbf{V}$ in $S O(3)$ leaves $d$ unchanged. If $K=C_{1}$ then $d=3-\operatorname{tr}\left(\overline{\mathbf{R}}^{2}\right)$, where $\overline{\mathbf{R}}=(\overline{\mathbf{X}} \top \overline{\mathbf{X}})^{1 / 2}$ with $\overline{\mathbf{X}}=\overline{\mathbf{t}}$, the sample mean of $\mathbf{X}=\left(\mathbf{u}_{1}, \mathbf{u}_{2}, \mathbf{u}_{3}\right)$, as in Mardia and Jupp [30, p. 290]. If $K=C_{2}$ then

$$
d=2-n^{-2} \sum_{k=1}^{n} \sum_{\ell=1}^{n}\left\{\mathbf{u}_{k 0}^{\top} \mathbf{u}_{\ell 0}+\left(\mathbf{u}_{k 1}^{\top} \mathbf{u}_{\ell 1}\right)^{2}\right\},
$$

where $\left[\mathbf{U}_{\mathbf{k}}\right]$ is represented by the $C_{2}$-frame $\left(\mathbf{u}_{k 0}, \pm \mathbf{u}_{k 1}\right)$ for $k \in\{1, \ldots, n\}$. If $K=D_{2}$ then $d=d_{1}$, where $d_{1}$ is one of the measures of dispersion defined in Arnold and Jupp [1, Section 2.3].

\section{Tests of uniformity}

\subsection{A simple test}

The uniform distribution on $S O(3) / K$ is the unique distribution that is invariant under the action of $S O(3)$ on $S O(3) / K$ in which $\mathbf{V}$ in $S O(3)$ maps $[\mathbf{U}]$ to [VU]. Since the embeddings $\mathbf{t}$ were chosen so that $\mathrm{E}\{\mathbf{t}([\mathbf{U}])\}=\mathbf{0}$ for $\mathbf{U}$ uniformly distributed on $S O(3) / K$, it is intuitively reasonable to reject uniformity if $\overline{\mathbf{t}}$ is far from $\mathbf{0}$, i.e., if $n\|\overline{\mathbf{t}}\|^{2}$ is large. 
Table 3: Inner products of transforms of symmetric frames.

\begin{tabular}{ll} 
Group, $K$ & Inner product \\
\hline$C_{1}$ & $\left\langle\mathbf{t}_{C_{1}}\left(\mathbf{u}_{1}, \mathbf{u}_{2}, \mathbf{u}_{3}\right), \mathbf{t}_{C_{1}}\left(\mathbf{v}_{1}, \mathbf{v}_{2}, \mathbf{v}_{3}\right)\right\rangle=\mathbf{u}_{1}^{\top} \mathbf{v}_{1}+\mathbf{u}_{2}^{\top} \mathbf{v}_{2}+\mathbf{u}_{3}^{\top} \mathbf{v}_{3}$ \\
$C_{2}$ & $\left\langle\mathbf{t}_{C_{2}}\left(\mathbf{u}_{0}, \pm \mathbf{u}_{1}\right), \mathbf{t}_{C_{2}}\left(\mathbf{v}_{0}, \pm \mathbf{v}_{1}\right)\right\rangle=\mathbf{u}_{0}^{\top} \mathbf{v}_{0}+\left(\mathbf{u}_{1}^{\top} \mathbf{v}_{1}\right)^{2}-1 / 3$ \\
$C_{r} \quad(r \geq 3)$ & \\
$r$ odd & $\left\langle\mathbf{t}_{C_{r}}\left(\left[\mathbf{u}_{1}, \ldots, \mathbf{u}_{r}\right]\right), \mathbf{t}_{C_{r}}\left(\left[\mathbf{v}_{1}, \ldots, \mathbf{v}_{r}\right]\right)\right\rangle=\mathbf{u}_{0}^{\top} \mathbf{v}_{0}+\sum_{i=1}^{r} \sum_{j=1}^{r}\left(\mathbf{u}_{i}^{\top} \mathbf{v}_{j}\right)^{r}$ \\
$r$ even & $\left\langle\mathbf{t}_{C_{r}}\left(\left[\mathbf{u}_{1}, \ldots, \mathbf{u}_{r}\right]\right), \mathbf{t}_{C_{r}}\left(\left[\mathbf{v}_{1}, \ldots, \mathbf{v}_{r}\right]\right)\right\rangle=\mathbf{u}_{0}^{\top} \mathbf{v}_{0}+\sum_{i=1}^{r} \sum_{j=1}^{r}\left(\mathbf{u}_{i}^{\top} \mathbf{v}_{j}\right)^{r}-r^{2} /(r+1)$ \\
$D_{2}$ & $\left\langle\mathbf{t}_{D_{2}}\left( \pm \mathbf{u}_{1}, \pm \mathbf{u}_{2}\right), \mathbf{t}_{D_{2}}\left( \pm \mathbf{v}_{1}, \pm \mathbf{v}_{2}\right)\right\rangle=\left(\mathbf{u}_{1}^{\top} \mathbf{v}_{1}\right)^{2}+\left(\mathbf{u}_{2}^{\top} \mathbf{v}_{2}\right)^{2}+\left(\mathbf{u}_{3}^{\top} \mathbf{v}_{3}\right)^{2}-1$ \\
$D_{r} \quad(r \geq 3)$ & \\
$r$ odd & $\left\langle\mathbf{t}_{D_{r}}\left(\left[\mathbf{u}_{1}, \ldots, \mathbf{u}_{r}\right]\right), \mathbf{t}_{D_{r}}\left(\left[\mathbf{v}_{1}, \ldots, \mathbf{v}_{r}\right]\right)\right\rangle=\sum_{i=1}^{r} \sum_{j=1}^{r}\left(\mathbf{u}_{i}^{\top} \mathbf{v}_{j}\right)^{r}$ \\
$r$ even & $\left\langle\mathbf{t}_{D_{r}}\left(\left[\mathbf{u}_{1}, \ldots, \mathbf{u}_{r}\right]\right), \mathbf{t}_{D_{r}}\left(\left[\mathbf{v}_{1}, \ldots, \mathbf{v}_{r}\right]\right)\right\rangle=\sum_{i=1}^{r} \sum_{j=1}^{r}\left(\mathbf{u}_{i}^{\top} \mathbf{v}_{j}\right)^{r}-r^{2} /(r+1)$ \\
$T$ & $\left\langle\mathbf{t}_{T}\left(\left\{\mathbf{u}_{1}, \mathbf{u}_{2}, \mathbf{u}_{3}, \mathbf{u}_{4}\right\}\right), \mathbf{t}_{T}\left(\left\{\mathbf{v}_{1}, \mathbf{v}_{2}, \mathbf{v}_{3}, \mathbf{v}_{4}\right\}\right)\right\rangle=\sum_{i=1}^{4} \sum_{j=1}^{4}\left(\mathbf{u}_{i}^{\top} \mathbf{v}_{j}\right)^{3}$ \\
$O$ & $\left\langle\mathbf{t}_{O}\left(\left\{ \pm \mathbf{u}_{1}, \pm \mathbf{u}_{2}, \pm \mathbf{u}_{3}\right\}\right), \mathbf{t}_{O}\left(\left\{ \pm \mathbf{v}_{1}, \pm \mathbf{v}_{2}, \pm \mathbf{v}_{3}\right\}\right)\right\rangle=\sum_{i=1}^{3} \sum_{j=1}^{3}\left(\mathbf{u}_{i}^{\top} \mathbf{v}_{j}\right)^{4}-9 / 5$ \\
$Y$ & $\left\langle\mathbf{t}_{Y}\left(\left\{ \pm \mathbf{u}_{1}, \ldots, \pm \mathbf{u}_{6}\right\}\right), \mathbf{t}_{Y}\left(\left\{ \pm \mathbf{v}_{1}, \ldots, \pm \mathbf{v}_{6}\right\}\right)\right\rangle=\sum_{i=1}^{6} \sum_{j=1}^{6}\left(\mathbf{u}_{i}^{\top} \mathbf{v}_{j}\right)^{10}-36 / 11$ \\
\hline
\end{tabular}

For $C_{r}$ with $r \geq 3, \mathbf{u}_{0}=\{\sin (2 \pi / r)\}^{-1} \mathbf{u}_{1} \times \mathbf{u}_{2}$. For $D_{2}, \mathbf{u}_{3}= \pm \mathbf{u}_{1} \times \mathbf{u}_{2}$.

For all sample sizes significance can be assessed using simulation from the uniform distribution. (Such simulation can be based on the fact that if $\mathbf{u}_{1}, \mathbf{u}_{2}$ are obtained by Gram-Schmidt orthonormalization of a pair of independent random vectors with the trivariate normal distribution with mean $\mathbf{0}$ and variance matrix $\mathbf{I}_{3}$ then, for any $K,\left[\left(\mathbf{u}_{1}, \mathbf{u}_{2}, \mathbf{u}_{1} \times \mathbf{u}_{2}\right)\right]$ is uniformly distributed on $S O(3) / K$.) For large samples, the following asymptotic result can be used.

Proposition 1. Given a random sample on $S O(3) / K$, define $S$ by

$$
S=\left(v / \rho^{2}\right) n\left\|\overline{\mathbf{t}}_{K}\right\|^{2}=n v\left(1-d / \rho^{2}\right)
$$

where $\rho^{2}$ and $d$ are given by (4) and (5), respectively, and $v$ is the dimension of $E$.

(i) For $K=C_{1}, D_{r}$ with $r \geq 2, T, O$ or $Y$, under uniformity, the asymptotic distribution of $S$ is $S \sim \chi_{v}^{2}$, as $n \rightarrow \infty$.

(ii) For $K=C_{2}$,

$$
S=(1 / 3) S_{R}+(2 / 15) S_{B},
$$

where $S_{R}=3 n \bar{R}^{2}$ is the Rayleigh statistic for uniformity of $\mathbf{u}_{0}$ and $S_{B}=(15 / 2) n\left\{\operatorname{tr}\left(\overline{\mathbf{T}}^{2}\right)-1 / 3\right\}$ is the Bingham statistic for uniformity of $\pm \mathbf{u}_{1}, \bar{R}$ being the mean resultant length of $\mathbf{u}_{0}$ and $\overline{\mathbf{T}}$ being the sample scatter matrix of $\pm \mathbf{u}_{1}$. Under uniformity, $S_{R}$ and $S_{B}$ are asymptotically independent with asymptotic distributions $\chi_{3}^{2}$ and $\chi_{5}^{2}$, respectively.

(iii) For $K=C_{r}$ with $r \geq 3$,

$$
\left(v_{C} / \rho_{C}^{2}\right) S=(1 / 3) S_{R}+\left(v_{D} / \rho_{D}^{2}\right) S_{D},
$$

where the subscripts $C$ and $D$ refer respectively to $C_{r}$-frames and the corresponding $D_{r}$-frames obtained by replacing the directed normal to the plane of a $C_{r}$-frame by the undirected normal, and $S_{R}=3 n \bar{R}^{2}$ is the Rayleigh statistic for uniformity of $\mathbf{u}_{0}$. Under uniformity, $S_{R}$ and $S_{D}$ are asymptotically independent with asymptotic distributions $\chi_{3}^{2}$ and $\chi_{v_{D}}^{2}$, respectively.

Values of $\rho^{2}$ and $v$ are given in Table 4. In the case $K=C_{1}, S$ is the Rayleigh statistic [30, p. 287] for testing uniformity on $S O(3)$. In the case $K=D_{2}, S$ is the statistic given in Arnold and Jupp [1, Section 3] for testing uniformity on $O(3) / \mathbb{Z}_{2}^{3}$.

Application of the randomization test of uniformity based on $S$ of (6) to the diopside data depicted in Figure 2 yields a $p$-value less than 0.001 , leading to decisive rejection of uniformity. 


\begin{tabular}{|c|c|c|}
\hline Group & $\rho^{2}$ & $v$ \\
\hline$C_{1}$ & 3 & 9 \\
\hline$C_{2}$ & $5 / 3$ & 8 \\
\hline$C_{r} \quad(r \geq 3)$ & & \\
\hline$r$ odd & $1+2^{1-r} r^{2}$ & $(r+2)(r+1) / 2+3$ \\
\hline$r$ even & $1+r^{2} 2^{1-r}\left\{1+2^{-1}\left(\begin{array}{c}r \\
r / 2\end{array}\right)\right\}-r^{2} /(r+1)$ & $(r+2)(r+1) / 2+3$ \\
\hline & 2 & 10 \\
\hline$D_{r} \quad(r \geq 3)$ & & \\
\hline$r$ odd & $r^{2} 2^{1-r}\left(1+2^{-1(r)} r\right) l-r^{2}(r+1)$ & $(r+2)(r+1) / 2$ \\
\hline $\begin{array}{l}r \text { even } \\
T\end{array}$ & $\begin{array}{c}r^{2} 2^{1-r}\left\{1+2^{-1}\left(\begin{array}{c}r \\
r / 2\end{array}\right)\right\}-r^{2} /(r+1) \\
32 / 9\end{array}$ & $(r+2)(r+1) / 2$ \\
\hline$O$ & $6 / 5$ & 15 \\
\hline$Y$ & $18816 / 6875$ & 66 \\
\hline
\end{tabular}

\subsection{Tests of other hypotheses using the embedding approach}

Permutational multi-sample tests, tests of symmetry, tests of independence, and goodness-of-fit tests for symmetric frames can be obtained by applying the machinery of Wellner [48], Jupp and Spurr [22], Jupp and Spurr [23], and Jupp [21], respectively, to the embedding t. These tests of independence are considered in Section 7.1.

\subsection{Some consistent tests of uniformity based on the averaging approach}

The test of uniformity based on $S$ is consistent only against alternatives for which $\mathrm{E}\{\mathbf{t}([\mathbf{U}])\}$ is non-zero. In principle, the embedding approach can be used to obtain tests of uniformity on $S O(3) / K$ that are consistent against all alternatives. However, it is difficult to obtain explicit expressions for the appropriate embedding, and so it is easier to take the averaging approach. More precisely, tests of uniformity on $S O(3) / K$ that are consistent against all alternatives can be obtained as follows by averaging over $K$ Prentice's generalization to $\mathbb{R} P^{3}$ of Giné's [12] $G_{n}$ test of uniformity; see Prentice [37, Section 4] and Mardia and Jupp [30, Section 13.2.2]. Given $\left[\mathbf{U}_{1}\right], \ldots,\left[\mathbf{U}_{n}\right]$ in $S O(3) / K$, with representatives $\mathbf{U}_{1}, \ldots, \mathbf{U}_{n}$ in $S O(3)$, put

$$
T_{G}=-\sum_{i=1}^{n} \sum_{j=1}^{n} \sum_{\mathbf{R} \in K}\left\{3-\operatorname{tr}\left(\mathbf{U}_{i}^{\top} \mathbf{U}_{j} \mathbf{R}\right)\right\}^{1 / 2},
$$

cf. the construction in Jupp and Spurr [22, Section 2]. Uniformity is rejected if $T_{G}$ is large compared with the randomization distribution obtained by replacing $\mathbf{U}_{1}, \ldots, \mathbf{U}_{n}$ by $\mathbf{R}_{1} \mathbf{U}_{1}, \ldots, \mathbf{R}_{n} \mathbf{U}_{n}$, where $\mathbf{R}_{1}, \ldots, \mathbf{R}_{n}$ are independent random rotations obtained from the uniform distribution on $S O(3)$. The orthorhombic case, $K=D_{2}$, is considered in Arnold and Jupp [1, Section 3]. It follows from Jupp and Spurr [22, Theorem 3.1] and the consistency of Giné's test on $\mathbb{R} P^{3}$ [30, p. 289] that the test based on $T_{G}$ is consistent against all alternatives. More general Sobolev statistics on $S O(3) / K$ can be obtained from Sobolev statistics on $S O(3)$ by averaging over $K$, as in (7).

\section{Distributions on $S O(3) / K$}

\subsection{A general class of distributions}

The scalar product $\langle\mathbf{t}([\mathbf{U}]), \mathbf{t}([\mathbf{V}])\rangle$ provides a measure of closeness between $[\mathbf{U}]$ and $[\mathbf{V}]$, and so an appealing class of distributions consists of those with densities of the form

$$
f([\mathbf{U}] ;[\mathbf{M}], \kappa)=g(\langle\mathbf{t}([\mathbf{U}]), \mathbf{t}([\mathbf{M}])\rangle ; \kappa),
$$

where $g(\cdot ; \kappa)$ is a suitable known function and $[\mathbf{M}] \in S O(3) / K$. The parameter $[\mathbf{M}]$ measures location and $\kappa$ measures concentration. If $g(\cdot ; \kappa)$ is a strictly increasing function, as in (9) or [29] with $\kappa>0$, then the mode is [M]. 
In the case $K=C_{1}$, the densities (8) depend on $\mathbf{U}$ only through $\operatorname{tr}\left(\mathbf{U M}^{\top}\right)$ and the axes and the rotation angles of the random rotations are independent, with the axes being uniformly distributed. These distributions were introduced by Bingham et al. [5] under the name of uniform axis-random spin distributions and by Hielscher et al. [18] under the name of radially symmetric distributions. For $K \neq C_{1}$, elements of $S O(3) / K$ do not have well-defined axes and, in general, the distributions on $S O(3)$ with densities $\tilde{f}$ of the form $\tilde{f}(\mathbf{U})=f([\mathbf{U}] ;[\mathbf{M}], \kappa)$ do not have uniformly distributed axes.

Taking $g(x ; \kappa)$ proportional to $e^{\kappa x}$ in (8) gives the densities of the exponential form

$$
f([\mathbf{U}] ;[\mathbf{M}], \kappa)=c(\kappa)^{-1} \exp \{\kappa\langle\mathbf{t}([\mathbf{U}]), \mathbf{t}([\mathbf{M}])\rangle\} .
$$

For $\kappa>0$, the mode is $[\mathbf{M}]$ and the maximum likelihood estimate of $[\mathbf{M}]$ based on a random sample $\left[\mathbf{U}_{1}\right], \ldots,\left[\mathbf{U}_{n}\right]$ is the sample mean, $[\overline{\mathbf{U}}]$. Differentiation of (9) shows that (for $\kappa>0$ ) the maximum likelihood estimate, $\hat{\kappa}$, of $\kappa$ is given by $\tau(\hat{\kappa})=\langle\overline{\mathbf{t}}, \mathbf{t}([\overline{\mathbf{U}}])\rangle$, where $\tau(\kappa)=d \ln c(\kappa) / d \kappa$ and $\overline{\mathbf{t}}$ is the sample mean of $\mathbf{t}\left(\left[\mathbf{U}_{1}\right]\right), \ldots, \mathbf{t}\left(\left[\mathbf{U}_{n}\right]\right)$.

The family (9) is a curved exponential family and is a subfamily of the crystallographic exponential family introduced by Boogaart [6, Section 3.2]. For $K=C_{1}$, (9) is the density of the matrix Fisher distribution with parameter matrix $\kappa \mathbf{M}$ and $c(\kappa)={ }_{0} F_{1}\left(3 / 2,\left(\kappa^{2} / 4\right) \mathbf{I}_{3}\right)$ [30, Section 13.2.3]. For $K=D_{2}$, (9) is the density of the equal-concentration frame Watson distributions considered by Arnold and Jupp [1, Section 6.1]. Taking $g(x ; \kappa)$ proportional to $(1+x)^{\kappa}$ in (8) gives the densities of the form

$$
f([\mathbf{U}] ;[\mathbf{M}], \kappa)=c(\kappa)^{-1}\{1+\langle\mathbf{t}([\mathbf{U}]), \mathbf{t}([\mathbf{M}])\rangle\}^{\kappa} .
$$

For $K=C_{1}$, these densities are those of the de la Vallée Poussin distributions introduced by Schaeben [41], and, under the name of Cayley distributions, by León et al. [29].

Taking $g(x)=1+\kappa x$ with $0 \leq \kappa \leq \rho^{-2}$ in (8) gives the densities

$$
f([\mathbf{U}] ;[\mathbf{M}], \kappa)=1+\kappa\langle\mathbf{t}([\mathbf{U}]), \mathbf{t}([\mathbf{M}])\rangle
$$

of the frame cardioid distributions, which are analogous to the cardioid distributions on the circle [30, Section 3.5.5]. Useful estimators of $[\mathbf{M}]$ and $\kappa$ in (11) are the moment estimators, $[\hat{\mathbf{M}}]$ and $\hat{\kappa}$, where $[\hat{\mathbf{M}}]$ is the sample mean defined in Section 3.2 and $\hat{\kappa}=\left(v / \rho^{3}\right)\|\overline{\mathbf{t}}\|$.

Distributions on $S O(3) / K$ can be identified with distributions on $S O(3)$ that are invariant under the action of $K$. One way of generating such distributions is to average a given distribution on $S O(3)$ over $K$, as in (1). This averaging construction has been used by Walsh et al. [47] in the orthorhombic case, Du et al. [11] and Chen et al. [9] in the cubic case, and by Matthies [31], Gorelova et al. [13] and Niezgoda et al. [35] in the general crystallographic case. Because the parameters of the distributions (8) are readily interpretable and the distributions (9), being exponential models, have pleasant inferential properties, we find these models more useful than many models obtained by averaging over $K$, especially as the latter can be quite demanding numerically.

\subsection{Concentrated distributions}

A standard coordinate system on $S O(3)$ is given by the inverse of a restriction of the exponential map $\mathbf{S} \mapsto$ $\sum_{k=0}^{\infty}(k !)^{-1} \mathbf{S}^{k}$ from the space of skew-symmetric $3 \times 3$ matrices to $S O(3)$. This can be modified to provide coordinate systems on $S O(3) / K$. Let $[\mathbf{M}]$ be an element of $S O(3) / K$. There are neighborhoods $\mathcal{N}_{[\mathbf{M}]}$ of $[\mathbf{M}]$ in $S O(3) / K$ and $\mathcal{V}$ of $\mathbf{0}$ in $\mathbb{R}^{3}$ such that each $[\mathbf{U}]$ in $\mathcal{N}_{[\mathbf{M}]}$ can be written uniquely as $[\mathbf{U}]=[\mathbf{M} \exp \{\mathbf{A}(\mathbf{v})\}]$, where

$$
\mathbf{A}(\mathbf{v})=\left(\begin{array}{ccc}
o & -v_{3} & v_{2} \\
v_{3} & 0 & -v_{1} \\
-v_{2} & v_{1} & 0
\end{array}\right)
$$

with $\mathbf{v}=\left(v_{1}, v_{2}, v_{3}\right)^{\top}$ in $\mathcal{V}$. Define $p_{[\mathbf{M}]}$ from $\mathcal{N}_{[\mathbf{M}]}$ to $\mathcal{V}$ by $p_{[\mathbf{M}]}([\mathbf{U}])=\mathbf{v}$, where $[\mathbf{U}]=[\mathbf{M} \exp \{\mathbf{A}(\mathbf{v})\}]$. Then $p_{[\mathbf{M}]}$ is a coordinate system on $\mathcal{N}_{[\mathbf{M}]}$. Second-order Taylor expansion about $\mathbf{0}$ of [U] as a function of $\mathbf{v}$, together with some computer algebra, gives the high-concentration asymptotic distribution of [U].

Proposition 2. For $[\mathbf{U}]$ near $[\mathbf{M}]$ in $S O(3) / K$ put $[\mathbf{U}]=[\mathbf{M} \exp \{\mathbf{A}(\mathbf{v})\}]$ for $\mathbf{v}$ near $\mathbf{0}$ in $\mathbb{R}^{3}$. If $[\mathbf{U}]$ has density (9) with $\mathbf{t}=\mathbf{t}_{K}$ as in Table 2 then the asymptotic distribution of $\kappa^{1 / 2} \mathbf{v}$ as $\kappa \rightarrow \infty$ is normal with mean $\mathbf{0}$ and variance $\mathbf{\Sigma}$, where $\boldsymbol{\Sigma}$ is given in Table 5. If $[\mathbf{U}]$ has density (10) with $\mathbf{t}=\mathbf{t}_{K}$ then $(\kappa / 2)^{1 / 2} \mathbf{v}$ has this asymptotic distribution. 
Table 5: High-concentration asymptotic variance, $\boldsymbol{\Sigma}$, of $\kappa^{1 / 2} \mathbf{v}$.

\begin{tabular}{|c|c|}
\hline Group & $\Sigma$ \\
\hline$C_{1}$ & $(1 / 2) \mathbf{I}_{3}$ \\
\hline$C_{2}$ & $\operatorname{diag}(1 / 2,1 / 4,1 / 6)$ \\
\hline$C_{r} \quad(r \geq 3)$ & $\operatorname{diag}\left[\left(1+r A_{r}\right)^{-1},\left(1+r A_{r}\right)^{-1},\left\{2 r A_{r}-r(r-1) A_{r-1}\right\}^{-1}\right]$ \\
\hline$D_{2}$ & $(1 / 4) \mathbf{I}_{3}$ \\
\hline$D_{r} \quad(r \geq 3)$ & $\operatorname{diag}\left[\left(r A_{r}\right)^{-1},\left(r A_{r}\right)^{-1},\left\{r A_{r}-r(r-1) A_{r-1}\right\}^{-1}\right]$ \\
\hline$T$ & $0.070 \mathbf{I}_{3}$ \\
\hline$O$ & $(1 / 8) \mathbf{I}_{3}$ \\
\hline$Y$ & $0.026 \mathbf{I}_{3}$ \\
\hline
\end{tabular}

For $C_{r}$ and $D_{r}, v_{3}$ is the component of $\mathbf{v}$ normal to the plane of $\mathbf{u}_{1}, \ldots, \mathbf{u}_{r}$ and $A_{r}=\sum_{k=1}^{r} \cos (k 2 \pi / r)^{r}$. The coefficients of $\mathbf{I}_{3}$ in $\boldsymbol{\Sigma}$ for $K=T, Y$ are rounded to 3 decimal places.

\section{Tests of location}

\subsection{One-sample tests}

Let $[\mathbf{M}]$ be an element of $S O(3) / K$ which is some measure of location of a distribution on $S O(3) / K$. There are various tests of the null hypothesis that $[\mathbf{M}]=\left[\mathbf{M}_{0}\right]$, where $\left[\mathbf{M}_{0}\right]$ is a given element of $S O(3) / K$. The case $K=D_{2}$ was considered in Arnold and Jupp [1, Section 8].

For $K=C_{r}$ with $r \geq 2$ or $D_{2}$, permutation tests can be based on the following symmetries of $S O(3) / K$. For $\mathbf{R}$ in $K$, define $\rho_{\left[\mathbf{M}_{0}\right]}(\mathbf{R})$ as the transformation that takes $[\mathbf{U}]$ to $\rho_{\left[\mathbf{M}_{0}\right]}(\mathbf{R})[\mathbf{U}]=\left[\mathbf{V R V} \mathbf{V}^{\top} \mathbf{U}\right]$, where $\mathbf{V}$ is any element of $\left[\mathbf{M}_{0}\right]$, i.e., $\mathbf{V}=\mathbf{M}_{0} \mathbf{W}$ for some $\mathbf{W}$ in $K$. Then $\rho_{\left[\mathbf{M}_{0}\right]}(\mathbf{R})$ is well-defined and preserves $\left[\mathbf{M}_{0}\right]$. For $K=C_{2},\left[\mathbf{M}_{0}\right]$ can be represented by a $C_{2}$-frame $\left(\mathbf{m}_{0}, \pm \mathbf{m}_{1}\right)$. (If the standard $C_{2}$-frame is taken as $\left(\mathbf{e}_{0}, \pm \mathbf{e}_{1}\right)$, where $\left(\mathbf{e}_{0}, \mathbf{e}_{1}, \mathbf{e}_{2}\right)$ is some specified orthonormal basis of $\mathbb{R}^{3}$, then one possibility for $\mathbf{M}_{0}$ is $\mathbf{M}_{0}=\left(\mathbf{m}_{0}, \mathbf{m}_{1}, \mathbf{m}_{0} \times \mathbf{m}_{1}\right)$ and $C_{2}$ can be identified with $\left\{\mathbf{I}, \mathbf{M}_{0} \mathbf{R}_{\pi} \mathbf{M}_{0}^{\top}\right\}$, where $\mathbf{R}_{\pi}=\operatorname{diag}(1,-1,-1)$.

For a sample summarized by the sample mean $\overline{\mathbf{t}}$ of $\mathbf{t}$, an appealing measure of the squared distance between the sample and $\left[\mathbf{M}_{0}\right]$ is $\left\|\overline{\mathbf{t}}-\mathbf{t}\left(\left[\mathbf{M}_{0}\right]\right)\right\|^{2}$. It is appropriate to reject the null hypothesis for large values of $\left\|\overline{\mathbf{t}}-\mathbf{t}\left(\left[\mathbf{M}_{0}\right]\right)\right\|^{2}$. If the distribution of $[\mathbf{U}]$ is symmetric under $\rho_{\left[\mathbf{M}_{0}\right]}$ then significance can be assessed by comparing the observed value of $\left\|\overline{\mathbf{t}}-\mathbf{t}\left(\left[\mathbf{M}_{0}\right]\right)\right\|^{2}$ with its randomization distribution, which can be obtained by replacing $\left[\mathbf{U}_{1}\right], \ldots,\left[\mathbf{U}_{n}\right]$ by $\rho_{\left[\mathbf{M}_{0}\right]}\left(\mathbf{R}_{1}\right)\left[\mathbf{U}_{1}\right], \ldots, \rho_{\left[\mathbf{M}_{0}\right]}\left(\mathbf{R}_{n}\right)\left[\mathbf{U}_{n}\right]$, where $\mathbf{R}_{1}, \ldots, \mathbf{R}_{n}$ are independent and distributed uniformly on $K$.

If $\left[\mathbf{U}_{1}\right], \ldots,\left[\mathbf{U}_{n}\right]$ is a sample from a concentrated distribution with density (9) and mode $[\mathbf{M}]$ then it is sensible to test $H_{0}:[\mathbf{M}]=\left[\mathbf{M}_{0}\right]$ by applying Hotelling's 1-sample $T^{2}$ test to $p_{\left[\mathbf{M}_{0}\right]}\left(\left[\mathbf{U}_{1}\right]\right), \ldots, p_{\left[\mathbf{M}_{0}\right]}\left(\left[\mathbf{U}_{n}\right]\right)$, where $p_{\left[\mathbf{M}_{0}\right]}$ is the projection onto the tangent space given in Section 5.2.

\subsection{Two-sample tests}

Suppose that two independent random samples $\left[\mathbf{U}_{1}\right], \ldots,\left[\mathbf{U}_{n}\right]$ and $\left[\mathbf{V}_{1}\right], \ldots,\left[\mathbf{V}_{m}\right]$ on $S O(3) / K$ are summarized by the sample means $\overline{\mathbf{t}}_{1}$ and $\overline{\mathbf{t}}_{2}$ of $\mathbf{t}\left(\left[\mathbf{U}_{1}\right]\right), \ldots, \mathbf{t}\left(\left[\mathbf{U}_{n}\right]\right)$ and $\mathbf{t}\left(\left[\mathbf{V}_{1}\right]\right), \ldots, \mathbf{t}\left(\left[\mathbf{V}_{m}\right]\right)$. Then the squared distance between the two samples can be measured by $\left\|\overline{\mathbf{t}}_{1}-\overline{\mathbf{t}}_{2}\right\|^{2}$. It is appropriate to reject the null hypothesis that the parent populations are the same if $\left\|\overline{\mathbf{t}}_{1}-\overline{\mathbf{t}}_{2}\right\|^{2}$ is large. Significance can be assessed by comparing the observed value of $\left\|\overline{\mathbf{t}}_{1}-\overline{\mathbf{t}}_{2}\right\|^{2}$ with its randomization distribution, obtained by sampling from the potential values corresponding to the partitions of the combined sample into samples of sizes $n$ and $m$.

Suppose that $\left[\mathbf{U}_{1}\right], \ldots,\left[\mathbf{U}_{n}\right]$ and $\left[\mathbf{V}_{1}\right], \ldots,\left[\mathbf{V}_{m}\right]$ are samples from concentrated distributions with density (9) on $S O(3) / K$. Let $[\dot{\mathbf{M}}]$ be the maximum likelihood estimate of the mode $[\mathbf{M}]$ under the null hypothesis that the parent populations are the same. Then the null hypothesis can be tested by applying Hotelling's 2-sample $T^{2}$ test to $p_{[\dot{\mathbf{M}}]}\left(\left[\mathbf{U}_{1}\right]\right), \ldots, p_{[\dot{\mathbf{M}}]}\left(\left[\mathbf{U}_{n}\right]\right)$ and $p_{[\dot{\mathbf{M}}]}\left(\left[\mathbf{V}_{1}\right]\right), \ldots, p_{[\dot{\mathbf{M}}]}\left(\left[\mathbf{V}_{m}\right]\right)$, where $p_{[\dot{\mathbf{M}}]}$ is the projection onto the tangent space given in Section 5.2.

The stereonets in Figure 3 show the $\mathbf{u}_{0}$ directions and $\pm \mathbf{u}_{1}$ axes given by the orientations of 34 diopside crystals from one region of a specimen and 37 diopside crystals from another region. The two-sample permutation test above 

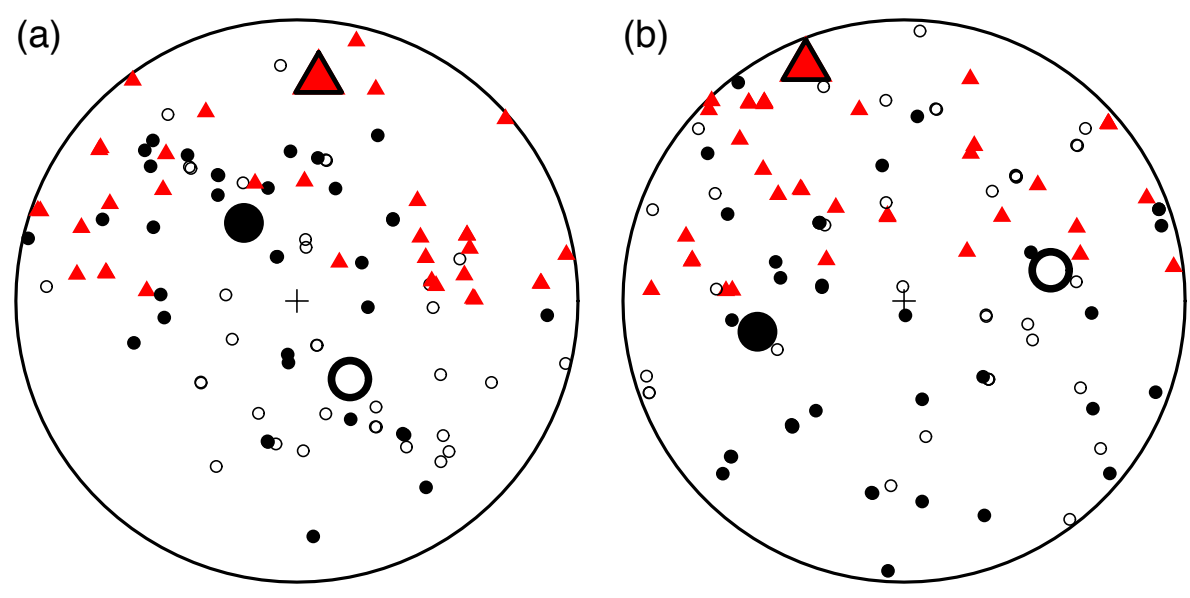

Figure 3: $C_{2}$ symmetry. Stereonets of $\mathbf{u}_{0}$ vectors, shown as red triangles, and $\pm \mathbf{u}_{1}$ axes, shown as circles, given by orientations of two samples of diopside crystals. Each disc is a stereographic projection of these axes and vectors, showing the whole of the sphere, so that each axis appears twice. (a): 34 orientations from one region of a specimen. (b): 37 orientations from another region. The sample means are shown as large symbols.

yields a $p$-value of 0.089 for equality of the populations of the orientations in the two regions, so the hypothesis of equality is not rejected.

\section{Independence, regression and misorientation}

\subsection{Independence}

Let $\mathbf{t}_{j}: S O(3) / K_{j} \rightarrow E$, for $j \in\{1,2\}$, be equivariant functions into some common inner-product space $E$ such that $\mathbf{t}_{j}([\mathbf{U}])$ has expectation $\mathbf{0}$ if $[\mathbf{U}]$ is uniformly distributed on $S O(3) / K_{j}$. Then association of random variables [U] on $S O(3) / K_{1}$ and $[\mathbf{V}]$ on $S O(3) / K_{2}$ can be measured in terms of association of $\mathbf{t}_{1}([\mathbf{U}])$ and $\mathbf{t}_{2}([\mathbf{V}])$.

The general approach of Jupp and Spurr [23] leads to the following test of independence. Given pairs $\left(\left[\mathbf{U}_{1}\right],\left[\mathbf{V}_{1}\right]\right)$, $\ldots,\left(\left[\mathbf{U}_{n}\right],\left[\mathbf{V}_{n}\right]\right)$ in $S O(3) / K_{1} \times S O(3) / K_{2}$, independence of $\mathbf{U}$ and $\mathbf{V}$ is rejected for large values of

$$
\sum_{i=1}^{n} \sum_{j=1}^{n}\left\langle\mathbf{t}_{1}\left(\left[\mathbf{U}_{i}\right]\right), \mathbf{t}_{1}\left(\left[\mathbf{U}_{j}\right]\right)\right\rangle\left\langle\mathbf{t}_{2}\left(\left[\mathbf{V}_{i}\right]\right), \mathbf{t}_{2}\left(\left[\mathbf{V}_{j}\right]\right)\right\rangle
$$

The observed value of this statistic is compared with the randomization distribution given $\left[\mathbf{U}_{1}\right], \ldots,\left[\mathbf{U}_{n}\right],\left[\mathbf{V}_{1}\right], \ldots$, $\left[\mathbf{V}_{n}\right]$. An alternative randomization test rejects independence for large values of the correlation coefficient $r$ defined in (14). For $K_{1}=K_{2}=C_{1}$ and $\mathbf{t}_{1}=\mathbf{t}_{2}=\mathbf{t}_{C_{1}}$ of Table 2, this is one of the tests considered by Rivest and Chang [40].

\subsection{Regression}

A reasonable model for homoscedastic regression of $[\mathbf{V}]$ in $S O(3) / K_{2}$ on $[\mathbf{U}]$ in $S O(3) / K_{1}$ has regression function $[\mathbf{U}] \mapsto[\mathbf{A U}]$ for some $\mathbf{A}$ in $S O(3)$ and error distribution that is a mild generalization of (9), so that the density of [V] given $[\mathbf{U}]$ is

$$
f([\mathbf{V}] \mid[\mathbf{U}] ; \mathbf{A}, \kappa)=c(\kappa)^{-1} \exp \left\{\kappa\left\langle\mathbf{t}_{2}([\mathbf{V}]), \mathbf{t}_{1}([\mathbf{A U}])\right\rangle,\right.
$$

where $\mathbf{t}_{1}$ and $\mathbf{t}_{2}$ map $S O(3) / K_{1}$ and $S O(3) / K_{2}$ into the same inner-product space $E$. For $K_{1}=K_{2}=K, \mathbf{t}_{1}$ and $\mathbf{t}_{2}$ can be taken as $\mathbf{t}_{K}$ of Table 2. If, further, $K=C_{1}$ then model (12) is a generalization of the spherical regression model of Chang [7]. It is the submodel $\mathbf{A}_{2}=\mathbf{I}_{3}$ of the models with regression function $\mathbf{U} \mapsto \mathbf{A} \mathbf{U} \mathbf{A}_{2}$ that were introduced by Prentice [38] and explored by Chang and Rivest [8] and Rivest and Chang [40]. For $K_{1} \neq C_{1}$, it is not possible in 
general to extend the model (12) to have regression function of the form [U] $\mapsto\left[\mathbf{A U A}_{2}\right]$. If $K_{1} \neq K_{2}$ then it is sensible to take $E=L^{2}\{S O(3)\}$ and $\mathbf{t}_{j}([\mathbf{U}])=\left|K_{j}\right|^{-1} \sum_{\mathbf{R} \in K_{j}} \mathbf{t}_{j 0}(\mathbf{U R})$ for $j \in\{1,2\}$, where $\mathbf{t}_{10}, \mathbf{t}_{20}: S O(3) \rightarrow L^{2}\{S O(3)\}$ are embeddings of the type used in the Hilbert space approach [30, Sections 10.8, 13.2.2] to Sobolev tests of uniformity. (Thus $\mathbf{t}_{j}$ is obtained using the averaging construction described in the second paragraph of Section 3.1.) If $K_{1}=C_{1}$ then a useful variant of (12) has

$$
f\left([\mathbf{V}] \mid \mathbf{U} ; \mathbf{A},\left[\mathbf{A}_{2}\right], \kappa\right)=c(\kappa)^{-1} \exp \left\{\kappa\left\langle\mathbf{t}_{2}([\mathbf{V}]), \mathbf{t}_{2}\left(\left[\mathbf{A U} \mathbf{A}_{2}\right]\right)\right\rangle\right\}
$$

with $\mathbf{A}$ in $S O(3)$ and $\left[\mathbf{A}_{2}\right]$ in $S O(3) / K_{2}$. The regression function is $\mathbf{U} \mapsto\left[\mathbf{A} \mathbf{U} \mathbf{A}_{2}\right]$.

For $\kappa>0$, the maximum likelihood estimate of $\mathbf{A}$ in (12) is

$$
\hat{\mathbf{A}}=\arg \max _{\mathbf{A} \in S O(3)} \sum_{i=1}^{n}\left\langle\mathbf{t}_{2}\left(\left[\mathbf{V}_{i}\right]\right), \mathbf{t}_{1}\left(\left[\mathbf{A} \mathbf{U}_{i}\right]\right)\right\rangle .
$$

In general, $\hat{\mathbf{A}}$ is a well-defined element of $S O(3)$, rather than an element of some quotient.

Put $\rho_{12}=\max _{\mathbf{U} \in S O(3)}\left\langle\mathbf{t}_{1}([\mathbf{U}]), \mathbf{t}_{2}\left(\left[\mathbf{I}_{3}\right]\right)\right\rangle$. If $\rho_{12}>0$ then define

$$
r=\frac{1}{n \rho_{12}} \sum_{i=1}^{n}\left\langle\mathbf{t}_{1}\left(\left[\hat{\mathbf{A}} \mathbf{U}_{i}\right]\right), \mathbf{t}_{2}\left(\left[\mathbf{V}_{i}\right]\right)\right\rangle,
$$

where $\hat{\mathbf{A}}$ is given by (13). Then $r \in[-1,1]$ and $r$ can be regarded as a form of uncorrected sample correlation of [U] and [V]. If $K_{1}=K_{2}=D_{2}, n=1$ and $\mathbf{t}_{1}=\mathbf{t}_{2}=\mathbf{t}$ is defined by $\mathbf{t}([\mathbf{U}])=\mathbf{U} \operatorname{diag}(1,0,-1) \mathbf{U}^{\top}$ then $r=\cos \omega$, where $\omega$ is the misorientation angle for $D_{2}$ introduced by Tape and Tape [45]. Application of Proposition 2 to the decomposition

$$
\begin{aligned}
\sum_{i=1}^{n}\left\{\rho^{2}-\left\langle\mathbf{t}\left(\left[\mathbf{I}_{3}\right]\right), \mathbf{t}\left(\left[\mathbf{V}_{i}^{\top} \mathbf{A} \mathbf{U}_{i}\right]\right)\right\rangle\right\}=\sum_{i=1}^{n}\left\{\rho^{2}-\left\langle\mathbf{t}\left(\left[\mathbf{I}_{3}\right]\right), \mathbf{t}\left(\left[\mathbf{V}_{i}^{\top} \hat{\mathbf{A}} \mathbf{U}_{i}\right]\right)\right\rangle\right\} & \\
& +\sum_{i=1}^{n}\left\{\left\langle\mathbf{t}\left(\left[\mathbf{I}_{3}\right]\right), \mathbf{t}\left(\left[\mathbf{V}_{i}^{\top} \hat{\mathbf{A}} \mathbf{U}_{i}\right]\right)\right\rangle-\left\langle\mathbf{t}\left(\left[\mathbf{I}_{3}\right]\right), \mathbf{t}\left(\left[\mathbf{V}_{i}^{\top} \mathbf{A} \mathbf{U}_{i}\right]\right)\right\rangle\right\}
\end{aligned}
$$

gives the following high-concentration asymptotic distributions.

Proposition 3. For $\left(\mathbf{U}_{1}, \mathbf{V}_{1}\right), \ldots,\left(\mathbf{U}_{n}, \mathbf{V}_{n}\right)$ from model (12),

(i) Asymptotically, for large $\kappa$,

$$
\begin{aligned}
2 \kappa \sum_{i=1}^{n}\left\{\rho^{2}-\left\langle\mathbf{t}\left(\left[\mathbf{I}_{3}\right]\right), \mathbf{t}\left(\left[\mathbf{V}_{i}^{\top} \mathbf{A} \mathbf{U}_{i}\right]\right)\right\rangle\right\} & \sim \chi_{3 n}^{2}, \\
2 \kappa \sum_{i=1}^{n}\left\{\rho^{2}-\left\langle\mathbf{t}\left(\left[\mathbf{I}_{3}\right]\right), \mathbf{t}\left(\left[\mathbf{V}_{i}^{\top} \hat{\mathbf{A}} \mathbf{U}_{i}\right]\right)\right\rangle\right\} & \sim \chi_{3(n-1)}^{2}, \\
2 \kappa \sum_{i=1}^{n}\left\{\left\langle\mathbf{t}\left(\left[\mathbf{I}_{3}\right]\right), \mathbf{t}\left(\left[\mathbf{V}_{i}^{\top} \hat{\mathbf{A}} \mathbf{U}_{i}\right]\right)\right\rangle-\left\langle\mathbf{t}\left(\left[\mathbf{I}_{3}\right]\right), \mathbf{t}\left(\left[\mathbf{V}_{i}^{\top} \mathbf{A} \mathbf{U}_{i}\right]\right)\right\rangle\right\} & \sim \chi_{3}^{2} .
\end{aligned}
$$

and the quantities in (17) and (18) are asymptotically independent.

(ii) An approximate high-concentration $100 \times(1-\alpha) \%$ confidence region for $\mathbf{A}$ is

$$
\left\{\mathbf{A}: 2 \hat{\kappa} \sum_{i=1}^{n}\left\{\left\langle\mathbf{t}\left(\left[\mathbf{I}_{3}\right]\right), \mathbf{t}\left(\left[\mathbf{V}_{i}^{\top} \hat{\mathbf{A}} \mathbf{U}_{i}\right]\right)\right\rangle-\left\langle\mathbf{t}\left(\left[\mathbf{I}_{3}\right]\right), \mathbf{t}\left(\left[\mathbf{V}_{i}^{\top} \mathbf{A} \mathbf{U}_{i}\right]\right)\right\rangle\right\}<\chi_{3 ; \alpha}^{2}\right\} .
$$




\subsection{Misorientation}

The relationship between ambiguous rotations $[\mathbf{U}]$ in $S O(3) / K_{1}$ and $[\mathbf{V}]$ in $S O(3) / K_{2}$ can be described by the misorientation, which is an element of the double coset space $K_{1} \backslash S O(3) / K_{2}$. Since [U] and [V] are images of $\mathbf{U R}_{1}$ and $\mathbf{V} \mathbf{R}_{2}$ in $S O$ (3) for any $\mathbf{R}_{1}$ in $K_{1}$ and $\mathbf{R}_{2}$ in $K_{2},\left(\mathbf{U R}_{1}\right)^{\top} \mathbf{V} \mathbf{R}_{2}$ determines a well-defined element of $K_{1} \backslash S O(3) / K_{2}$. See Morawiec [33, p. 274]. In crystallography it is usual to identify $K_{1} \backslash S O(3) / K_{2}$ with an asymmetric domain, a neighbourhood of $\mathbf{0}$ in $\mathbb{R}^{3}$ that is in one-to-one correspondence, modulo null sets, with $K_{1} \backslash S O(3) / K_{2}$ under $\mathbf{v} \mapsto$ $\exp \{\mathbf{A}(\mathbf{v})\}$ followed by projection of $S O(3)$ to $K_{1} \backslash S O(3) / K_{2}$. Then the misorientation between $[\mathbf{U}]$ and $[\mathbf{V}]$ is taken as the element, $\mathbf{P}$, of the asymmetric domain that satisfies $\mathbf{V}=\mathbf{U P}$ and has smallest rotation angle among all such rotations in the domain. In the case in which the conditional distribution of $[\mathbf{V}]$ given $[\mathbf{U}]$ is uniform the distributions of the angle and axis of the misorientation are given in Morawiec [34, Chapter 7]. For general pairs $\left(\left[\mathbf{U}_{1}\right],\left[\mathbf{V}_{1}\right]\right), \ldots,\left(\left[\mathbf{U}_{n}\right],\left[\mathbf{V}_{n}\right]\right)$ in $S O(3) / K_{1} \times S O(3) / K_{2}$, we define the mean misorientation as the element $\hat{A}$ of $S O(3)$ defined in (13). An alternative definition of the mean misorientation is the element $\left(\hat{\mathbf{A}}_{1},\left[\hat{\mathbf{A}}_{2}\right]\right)$ of $S O(3) \times(K \backslash S O(3))$ that maximizes $\sum_{i} \max _{\mathbf{R}_{i} \in K}\left\langle\mathbf{t}_{1}\left(\left[\mathbf{A}_{1} \mathbf{U}_{i}\right]\right), \mathbf{t}_{2}\left(\left[\mathbf{V}_{i} \mathbf{R}_{i} \mathbf{A}_{2}\right]\right)\right\rangle$.

\section{Concluding remarks}

Suitable statistical models for objects with high degrees of symmetry have until now relied almost exclusively on the averaging approach to account for ambiguous rotations. This approach is cumbersome, since each observation contributes to the likelihood via a sum over a set of equivalent orientations. In contrast, the embedding approach creates structures which respect the relevant symmetry automatically, and it does so in a unified way for all finite symmetry groups.

By exploiting the embedding approach, this paper has placed the study of ambiguous rotations on a sound theoretical basis and has provided basic computable tools for the analysis of data of this form. The statistical tests and probability models that we have presented here form a first step towards the analysis of data on ambiguous rotations that arise in complicated contexts. For example, we anticipate that elaborations of the distributions presented in Section 5 will be required when observations are taken across sites in a crystal, where spatial dependence should be included. Non-parametric approaches, beyond those of the simple tests of uniformity and location presented in Section 4, will allow the modelling of a wider range of behaviours. Subsequent work will include settings in which more than one symmetry class is present, such as those considered from another point of view in [19].

Acknowledgements. We thank David Mainprice for providing the diopside data.

\section{Appendix: Proofs and calculations}

Some moments. If $\mathbf{u}$ is uniformly distributed on $S^{2}$ and $\mathbf{v}$ is a fixed unit vector then $\mathrm{E}\left\{\left(\mathbf{u}^{\top} \mathbf{v}\right)^{r}\right\}=\mathrm{E}\left(u_{1}^{r}\right)$, where $u_{1}$ is uniformly distributed on $[-1,1]$. It follows that $\left\langle\mathrm{E}\left[\otimes^{r} \mathbf{u}\right], \otimes^{r} \mathbf{v}\right\rangle=\mathrm{E}\left\{\left(\mathbf{u}^{\top} \mathbf{v}\right)^{r}\right\}=1 /(r+1)$ (for $r$ even) or 0 (for $r$ odd). Thus if $r$ is odd,

$$
\mathrm{E}\left(\otimes^{r} \mathbf{u}\right)=\mathbf{0} .
$$

If $r$ is even then

$$
\left\langle\operatorname{symm}\left(\otimes^{r / 2} \mathbf{I}_{3}\right), \otimes^{r} \mathbf{v}\right\rangle=1 .
$$

It follows from (A1) and (A2) that the $\mathbf{t}_{K}$ of Table 2 satisfy $\mathrm{E}\left\{\mathbf{t}_{K}([\mathbf{U}])\right\}=\mathbf{0}$ for $\mathbf{U}$ uniformly distributed on $S O(3) / K$.

Values of $\rho^{2}$ and $v$. The values of $\rho^{2}$ given in Table 4 are obtained from the inner products given in Table 2, together with some computer algebra for simplifying the summation needed when $K=D_{r}$ with $r \geq 3$.

For $K=C_{1}, C_{2}$ or $D_{2}$, calculation of $v$ (the dimension of the range of $\mathbf{t}_{K}$ ) is straightforward. Since the space of symmetric $r$-way arrays on $\mathbb{R}^{p}$ has dimension $\left(\begin{array}{c}p+r-1 \\ r\end{array}\right)$; see (7.20) in [14]. Taking $p=3$ shows that for $K=D_{r}(r \geq$ 3), $T, O$ and $Y$, the value of $v$ is that given in Table 4. Comparison of $\mathbf{t}_{C_{r}}$ and $\mathbf{t}_{D_{r}}$ shows that $v_{C_{r}}=v_{D_{r}}+3$. 
Injectivity of $\mathbf{t}_{K}$. For $K=C_{1}, C_{2}$ or $D_{2}$, injectivity follows easily from the form of $\mathbf{t}_{K}$.

Let $\left[\mathbf{v}_{1}, \ldots, \mathbf{v}_{r}\right]$ and $\left[\mathbf{w}_{1}, \ldots, \mathbf{w}_{r}\right]$ be two $D_{r}$-frames with $r \geq 3$. Put $V=\operatorname{span}\left\{\mathbf{v}_{1}, \ldots, \mathbf{v}_{r}\right\}$ and $W=\operatorname{span}\left\{\mathbf{w}_{1}, \ldots, \mathbf{w}_{r}\right\}$. Let $\mathbf{e}_{1}, \mathbf{e}_{2}, \mathbf{e}_{3}$ be an orthonormal basis of $\mathbb{R}^{3}$ with $\mathbf{e}_{1} \in V \cap W$ and $\mathbf{e}_{2} \in V$. Then, possibly after reversing the orders of $\mathbf{v}_{1}, \ldots, \mathbf{v}_{r}$ or of $\mathbf{w}_{1}, \ldots, \mathbf{w}_{r}$, we can find $\theta_{0}$ and $\delta$ such that, for all $j \in\{1, \ldots, r\}$,

$$
\mathbf{v}_{j}=\cos \left(\theta_{j}\right) \mathbf{e}_{1}+\sin \left(\theta_{j}\right) \mathbf{e}_{2}, \quad \mathbf{w}_{j}=\cos \left(\theta_{j}+\delta\right) \mathbf{e}_{1}+\cos (\alpha) \sin \left(\theta_{j}+\delta\right) \mathbf{e}_{2}+\sin (\alpha) \sin \left(\theta_{j}+\delta\right) \mathbf{e}_{3},
$$

where $\theta_{j}=\theta_{0}+j 2 \pi / r$ for $j \in\{1, \ldots, r\}$ and $\alpha$ (with $\left.0 \leq \alpha \leq \pi / 2\right)$ is the angle between $V$ and $W$. If $t_{D_{r}}\left(\left[v_{1}, \ldots, v_{r}\right]\right)=$ $t_{D_{r}}\left(\left[w_{1}, \ldots, w_{r}\right]\right)$ then expansion of $\sum_{j=1}^{r} \otimes^{r} \mathbf{v}_{j}$ and $\sum_{j=1}^{r} \otimes^{r} \mathbf{w}_{j}$ in terms of symmetric tensor products of $\mathbf{e}_{1}, \mathbf{e}_{2}, \mathbf{e}_{3}$ leads to

$$
r \exp \left\{i r \theta_{0}\right\}=\sum_{j=1}^{r}\left\{\cos \left(\theta_{j}\right)+i \sin \left(\theta_{j}\right)\right\}^{r}=\sum_{j=1}^{r}\left\{\cos \left(\theta_{j}+\delta\right)+i \cos (\alpha) \sin \left(\theta_{j}+\delta\right)\right\}^{r} .
$$

The triangle inequality yields $\alpha=0$ or $\pi$ and $\theta_{0}+\delta= \pm \theta_{0}$. Thus $\left[v_{1}, \ldots, v_{r}\right]=\left[w_{1}, \ldots, w_{r}\right]$.

Injectivity for $C_{r}(r \geq 3)$ follows from that for $D_{r}$. Injectivity for $T, O$ and $Y$ is established in a similar way but using computer algebra to handle the trigonometric sums that arise.

Proof of Proposition 1. Result (i) for $K=C_{1}$ and $K=D_{2}$ is given in [30, Section 13.2.2] and [1, Proposition 1], respectively. For general $K$ it follows from the multivariate Central Limit Theorem and the fact that, under uniformity, $\mathrm{E}\left\{\mathbf{t}_{K}([\mathbf{U}])\right\}=\mathbf{0}$ that the large-sample asymptotic null distribution of $n^{1 / 2} \overline{\mathbf{t}}_{K}$ is multivariate normal $\boldsymbol{N}_{v}(\mathbf{0}, \boldsymbol{\Sigma})$, where $\boldsymbol{\Sigma}$ is the variance of $\mathbf{t}_{K}([\mathbf{U}])$. For $K=D_{r}$ with $r \geq 3, T, O$ or $Y,\left[\mathbf{u}_{1}, \ldots, \mathbf{u}_{r}\right]$ uniformly distributed on $S O(3) / K$ and $\mathbf{v}$ a unit vector in $\mathbb{R}^{3}$,

$$
\left\langle\otimes^{r} \mathbf{v}, \boldsymbol{\Sigma} \otimes^{r} \mathbf{v}\right\rangle=\left\langle\otimes^{r} \mathbf{v}, \operatorname{var}\left(\mathbf{t}_{K}\left(\left[\mathbf{u}_{1}, \ldots, \mathbf{u}_{r}\right]\right)\right) \otimes^{r} \mathbf{v}\right\rangle=\operatorname{var}\left\{\sum_{i=1}^{k}\left(\mathbf{u}_{i}^{\top} \mathbf{v}\right)^{r}\right\} .
$$

Thus, for $\mathbf{U}$ in $S O(3),\left(\otimes^{r} \mathbf{U}\right) \mathbf{\Sigma}\left(\otimes^{r} \mathbf{U}\right)^{\top}=\boldsymbol{\Sigma}$, where $\otimes^{r} \mathbf{U}$ is defined by $\left(\otimes^{r} \mathbf{U}\right)\left(\mathbf{v}_{1} \otimes \cdots \otimes \mathbf{v}_{r}\right)=\left(\mathbf{U} \mathbf{v}_{1}\right) \otimes \cdots \otimes\left(\mathbf{U} \mathbf{v}_{r}\right)$.

Choose a unit vector, $\mathbf{v}_{0}$, in $\mathbb{R}^{3}$ and put $c=\left\langle\otimes^{r} \mathbf{v}_{0}, \boldsymbol{\Sigma} \otimes^{r} \mathbf{v}_{0}\right\rangle$. Define $Z=\left\{\mathbf{w} \in E:\|\mathbf{w}\|=1, \mathbf{w}^{\top}\left(\boldsymbol{\Sigma}-c \mathbf{I}_{v}\right) \mathbf{w}=0\right\}$, where $E$ is the appropriate inner-product space. Then $Z$ is non-empty and is mapped into itself by each $\otimes^{r} \mathbf{U}$. Given orthonormal vectors $\mathbf{e}_{1}, \mathbf{e}_{2}, \mathbf{e}_{3}$ in $\mathbb{R}^{3}$, consider the functions

$$
\theta \mapsto \otimes^{r}\left\{\cos (\theta) \mathbf{e}_{j}+\sin (\theta) \mathbf{e}_{k}\right\}
$$

(with $k \neq j$ ) of the unit circle into $E$. Linear independence of the trigonometric functions $\cos (\theta)^{r}, \ldots, \cos (\theta)^{i} \sin (\theta)^{r-i}$, $\ldots, \sin (\theta)^{r}$ leads to the result that $\mathbf{t}_{K}(S O(3) / K)$ is not contained in a proper subspace of $E$. Thus any eigenspace of $\boldsymbol{\Sigma}-c \mathbf{I}_{v}$ contains an element of $Z$, which shows that the corresponding eigenvalue is zero, and so $\boldsymbol{\Sigma}=c \mathbf{I}_{v}$. Taking the trace of $\Sigma$ and using (4) shows that $\rho^{2}=\operatorname{tr}(\boldsymbol{\Sigma})=c v$, from which part (i) follows. Parts (ii) and (iii) are simple calculations.

Proof of Proposition 2. For $\mathbf{v}$ near $\mathbf{0}$ in $\mathbb{R}^{3}$, the log density of [U] is, up to an additive constant, $\left.\kappa\langle\mathbf{t}(\exp \{\mathbf{A}(\mathbf{v})\}]), \mathbf{t}\left(\left[\mathbf{I}_{3}\right]\right)\right\rangle$. Some computer algebra then shows that the log density of $\kappa^{1 / 2} \mathbf{v}$ is, up to an additive constant,

$$
-(1 / 2)\left(\kappa^{1 / 2} \mathbf{v}\right)^{\top} \boldsymbol{\Sigma}^{-1}\left(\kappa^{1 / 2} \mathbf{v}\right)+O\left(\kappa^{-1 / 2}\right)
$$

where $\boldsymbol{\Sigma}$ is given in Table 5. The asymptotic distribution of $\kappa^{1 / 2} \mathbf{v}$ follows.

Proof of Proposition 3. By Proposition 2 if $\kappa$ is large then $2 \kappa\left\{\rho^{2}-\left\langle\mathbf{t}\left(\left[\mathbf{I}_{3}\right]\right), \mathbf{t}\left(\left[\mathbf{V}_{i}^{\top} \mathbf{A} \mathbf{U}_{i}\right]\right)\right\rangle\right\} \sim \chi_{3}^{2}$ for $i \in\{1, \ldots, n\}$. Summing this over $i$ gives (16). Multiplying (15) by $\kappa$ gives a decomposition in which, if $\kappa$ is large, each of the three terms is approximately quadratic in $\kappa^{1 / 2}(\hat{\mathbf{A}}-\mathbf{A})$. Cochran's theorem then yields part (i). Part (ii) follows from (18). 


\section{References}

[1] R. Arnold, P.E. Jupp, Statistics of orthogonal axial frames, Biometrika 100 (2013) 571-586.

[2] R. Arnold, J. Townend, A Bayesian approach to estimating tectonic stress from seismological data, Geophys. J. Int. 170 (2007) $1336-1356$.

[3] R. Bhattacharya, V. Patrangenaru, Large sample theory of intrinsic and extrinsic sample means on manifolds I, Ann. Statist. 31 (2003) 1-29.

[4] L. Bindi, P. Steinhardt, N. Yao, P.J. Lu, Icosahedrite, $\mathrm{Al}_{6} \mathrm{Cu}_{24} \mathrm{Fe}_{13}$, the first natural quasicrystal, Amer. Mineralogist 96 (2011) $928-31$.

[5] M.A. Bingham, D.J. Nordman, S.B. Vardeman, Modeling and inference for measured crystal orientations and a tractable class of symmetric distributions for rotations in three dimensions, J. Amer. Statist. Assoc. 104 (2009) 1385-97.

[6] K.G. van den Boogaart, Statistics for Individual Crystallographic Orientation Measurements, Shaker Verlag, Aachen, Germany, 2002.

[7] T. Chang, Spherical regression, Ann. Statist. 14 (1986) 907-924.

[8] T. Chang, L.-P. Rivest, M-estimation for location and regression models on Stiefel manifolds, Ann. Statist. 29 (2001) $784-814$.

[9] Y.-H. Chen, D. Wei, G. Newstad, M. DeGraef, J. Simmons, A. Hero, Parameter estimation in spherical symmetry groups, IEEE Signal Proc. Lett. 22 (2015) 1152-55.

[10] Y. Chikuse, P.E. Jupp, A test of uniformity on shape spaces, J. Mult. Anal. 88 (2004) 163-176.

[11] C. Du, D.J. Nordman, S.B. Vardeman, Bayesian inference for a new class of distributions on equivalence classes of 3-D orientations with applications to materials science, Technometrics 58 (2016) 214-224.

[12] M. Giné, E., Invariant tests for uniformity on compact Riemannian manifolds based on Sobolev norms, Ann. Statist. 3 (1975) $1243-1266$.

[13] S. Gorelova, H. Schaeben, R. Kawalla, Quantifying texture evolution during hot rolling of magnesium twin roll cast strip, Mater. Sci. Eng. A 602 (2014) 134-142.

[14] W.H. Greub, Multilinear Algebra, Springer, Berlin, 1967.

[15] T. Griffiths, G. Habler, R.Abart, Crystallographic orientation relationships in host-inclusion systems: New insights from large EBSD data sets, Amer. Mineralogist 101 (2016) 690-705.

[16] J.L. Hardebeck, Homogeneity of small-scale earthquake faulting, stress, and fault strength, Bull. Seismological Soc. America 96 (2006) 1675-1688.

[17] S.C. Harrison, Principles of Virus Structure, In: D.M. Knipe, P.M. Howley, J.L. Cohen, D.E. Griffin, R.A. Lamb, J.L. Martin, V.R. Racaniello, B. Roizman (Eds.) Fields Virology, 6th ed., Wolters Kluwer Health/Lippincott Williams and Wilkins, Philadelphia, 2013, pp. $52-86$.

[18] R. Hielscher, H. Schaeben, H. Siemes, Orientation distribution within a single hematite crystal, Math. Geosciences 42 (2010) $359-375$.

[19] M. Humbert ,F. Wagner, H. Moustahfid, C. Esling, Determination of the orientation of a parent $\beta$ grain from the orientations of the inherited $\alpha$ plates in the phase transformation from body-centred cubic to hexagonal close packed, J. Appl. Cryst. 28 (1995) 571-576.

[20] E.D. Jemmis, Overlap control and stability of polyhedral molecules. closo-carboranes, J. Am. Chem. Soc. 104 (1982) 7017-7020.

[21] P.E. Jupp, Sobolev tests of goodness of fit of distributions on compact Riemannian manifolds, Ann. Statist. 33 (2005) $2957-2966$.

[22] P.E. Jupp, B.D. Spurr, Sobolev tests for symmetry of directional data, Ann. Statist. 11 (1983) 1225-1231.

[23] P.E. Jupp, B.D. Spurr, Sobolev tests for independence of directions, Ann. Statist. 13 (1985) 1140-1155.

[24] S.M. Khalil, K.R. McClay, 3D geometry and kinematic evolution of extensional fault-related folds, NW Red Sea, Egypt, In: C. Childs, R.E. Holdsworth, C.A.-L. Jackson, T. Manzocchi, J.J. Walsh, G. Yielding (Eds.), The Geometry and Growth of Normal Faults, Geological Society of London, London, 2016.

[25] M.R. Koymans, C.G. Langereis, D. Pastor-Galán, D.J.J. van Hinsbergen, Paleomagnetism.org: An online multi-platform open source environment for paleomagnetic data analysis, Computers in Geosciences 93 (2016)127-137.

[26] K. Kunze, H. Schaeben, The Bingham distribution of quaternions and its spherical Radon transform in texture analysis, Math. Geology 36 (2004) 917-943.

[27] K. Kunze, H. Schaeben, Ideal patterns of crystallographic preferred orientation and their representation by the von Mises-Fisher matrix or Bingham quaternion distribution, Materials Science Forum $495-497$ (2005) 295-300.

[28] K. Lekadir, J. Hazrati-Marangalou, C. Hoogendoorn, Z. Taylor, B. van Rietbergen, A.F. Frang, Statistical estimation of femur microarchitecture using optimal shape and density predictors, J. Biomechanics 48 (2015) 598-603.

[29] C. León, J.-C. Massé, L.-P. Rivest, A statistical model for random rotations, J. Multivariate Anal. 97 (2006) $412-430$.

[30] K.V. Mardia, P.E. Jupp, Directional Statistics, Wiley, Chichester, 2000.

[31] S. Matthies, Aktuelle Probleme der quantitativen Texturanalyse, Akademie der Wissenschaften der DDR, Rossendorf, Germany, 1982.

[32] W. Miller, Symmetry Groups and their Applications, Academic Press, New York, 1972.

[33] A. Morawiec, Distributions of misorientation angles and misorientation axes for crystallites with different symmetries, Acta Crystallographica A 53 (1997) 273-285.

[34] A. Morawiec, Orientations and Rotations, Springer-Verlag, Berlin, 2004.

[35] S.R. Niezgoda, E.A. Magnuson, J. Glover, Symmetrized Bingham distribution for representing texture: Parameter estimation with respect to crystal and sample symmetries, J. Appl. Cryst. 49 (2016) 1315-1319.

[36] L.J. Pesonen, S.-A. Elming, S. Mertanen, S. Pisarevsky, M.S. D’Agrella-Filho, J.-G. Meert, P.W. Schmidt, N. Abrahamsen, G. Bylund, Palaeomagnetic configuration of continents during the Proterozoic, Tectonophysics 375 (2003) 289-324.

[37] M.J. Prentice, On invariant tests of uniformity for directions and orientations, Ann. Statist. 6 (1978) 169-176.

[38] M.J. Prentice, Spherical regression on matched pairs of orientation statistics, J. R. Statist. Soc. B 51 (1989) $241-248$.

[39] L.-P. Rivest, A correction for axis misalignment in the joint angle curves representing knee movement in gait analysis, J. Biomechanics 38 (2005) 1604-1611.

[40] L.-P. Rivest, T. Chang, Regression and correlation for $3 \times 3$ rotation matrices, Canad. J. Statist. 34 (2006) 187-202.

[41] H. Schaeben, A simple standard orientation density function: The hyperspherical de la Vallée Poussin kernel, Phys. Stat. Sol. (b) 200 (1997) 367-376.

[42] T. Seideman, The liquid-crystalline blue phases, Rep. Prog. Phys. 53 (1990) 659-705. 
[43] B. Spronck, R.T.A. Megens, K.D. Reesink, T. Delhaas, A method for three-dimensional quantification of vascular smooth muscle orientation: application in viable murine carotid arteries, Biomechanics and Modeling in Mechanobiology 15 (201 6) 41 9-432.

[44] S. Stein, M. Wysession, An Introduction to Seismology, Earthquakes and Earth Structure, Blackwell, Malden, MA, 2003.

[45] W. Tape, C. Tape, Angle between principal axes, Geophys. J. Internat. 191 (2012) 813-31.

[46] J.J. Villalaín, A.M. Casas-Sainz, R. Soto, Reconstruction of inverted sedimentary basins from syntectonic remagnetizations. A methodological proposal, In: E.L. Pueyo, F. Cifelli, A.J. Sussman, B. Oliva-Urcia (Eds.), Palaeomagnetism in Fold and Thrust Belts: New Perspectives, Geological Society of London, London, 2016, pp. 233-246.

[47] D. Walsh, R. Arnold, J. Townend, A Bayesian approach to determining and parameterising earthquake focal mechanisms, Geophys. J. Int. 176 (2009) 235-255.

[48] J.A. Wellner, Permutation tests for directional data, Ann. Statist. 7 (1979) 929-43. 